\title{
Localization Problem in Index Theory of Elliptic Operators
}

\author{
Vladimir Nazaikinskii* \\ Institute for Problems in Mechanics, Russian Academy of Sciences \\ pr. Vernadskogo 101-1, 117526 Moscow, Russia \\ e-mail: nazaikinskii@mtu-net.ru \\ Bert-Wolfgang Schulze \\ Institut für Mathematik, Universität Potsdam \\ Am Neuen Palais 10, 14469 Postdam \\ Postfach 601553, 14469 Potsdam, Germany \\ e-mail: schulze@math.uni-potsdam.de \\ Boris Sternin* \\ Department of Computational Mathematics and Cybernetics \\ Moscow State University, Vorob'evy Gory, 119899 Moscow, Russia \\ e-mail: sternine@mtu-net.ru
}

\begin{abstract}
This is a survey of recent results concerning the general index locality principle, associated surgery, and their applications to elliptic operators on smooth manifolds and manifolds with singularities as well as boundary value problems. The full version of the paper is submitted for publication in Russian Mathematical Surveys.
\end{abstract}

Keywords: elliptic operators, index theory, surgery, relative index, manifold with singularities.

2000 AMS classification: Primary 58J20, Secondary 58J30, 58J40, 19 K56

*Supported by the Russian Foundation for Basic Research under grants Nos. 99-01-01100, 99-01-01254, and 00-01-00161 and by the DAAD. 


\section{Contents}

Introduction $\quad 2$

1 The abstract locality principle $\quad 4$

1.1 Bottleneck spaces . . . . . . . . . . . . . . . . 4

1.2 Elliptic operators . . . . . . . . . . . . . . 7

1.3 The relative index theorem . . . . . . . . . . . . . 10

2 Localization in index theory on smooth manifolds 13

2.1 Surgery on compact manifolds . . . . . . . . . . . . . . 13

2.2 Surgery on noncompact manifolds . . . . . . . . . . . . 16

3 Localization for boundary value problems $\quad 18$

3.1 Some notation . . . . . . . . . . . . . . . . . 18

3.2 General boundary value problem . . . . . . . . . . . . . . 20

3.3 Model boundary value problems on the cylinder . . . . . . . . . . 22

3.4 The Agranovich-Dynin theorem . . . . . . . . . . . . . . . . . 24

3.5 The Agranovich theorem . . . . . . . . . . . . . . 26

3.6 The Bojarski theorem and its generalizations . . . . . . . . . . . . 27

3.7 Boundary value problems with symmetric conormal symbol . . . . . . . . 29

4 Localization in index theory on singular manifolds 30

4.1 Index of elliptic $\Psi$ DO . . . . . . . . . . . . . . . 30

4.2 The index of Fourier integral operators . . . . . . . . . . . . . 43

\section{Introduction}

In the construction of index formulas for elliptic operators on manifolds with boundary, singular manifolds, or noncompact manifolds with a special structure at infinity ("cylindrical ends"), the problem of separating index contributions from the "interior" part of the manifold an the boundary, singular points, or a neighborhood of infinity is often topical. Putting forward this problem is justified by the "locality" of the index. The fact that the index of an elliptic operator on a smooth compact manifold without boundary possesses some locality property was known in elliptic theory at least since the so-called "local index formulas" had emerged (e.g., see [15] and the papers cited therein). A local formula represents the index of an elliptic operator $D$ on a closed manifold $M$ in the form

$$
\text { ind } D=\int_{M} \alpha(x)
$$


where the "local density" $\alpha(x)$ at a point $x \in M$ depends only on a finite-order jet of the principal symbol $\sigma(D)$ in the fiber over $x$. A more careful consideration shows that locality property is actually a property not of the index itself, but of the relative index, i.e., the difference of indices of two operators differing on some subset $\widetilde{M} \subset M$ and coinciding on $M \backslash \widetilde{M}$. Namely, this difference is expressed as the integral over $\widetilde{M}$ of the difference of the corresponding local densities and hence is independent of the (common) structure of the two operators in question on $M \backslash \widetilde{M}$. For the case in which local index formulas like $(0.1)$ are not known a priori, the proof of the relative index locality is more complicated. For the case of Dirac operators on complete noncompact Riemannian manifolds the locality property was proved by Gromov and Lawson [16], whose result was later generalized in various directions (e.g., see [3]).

We consider a general functional-analytic model in which the locality principle is valid for the (relative) index. We point out that, in contrast with the above reasoning for closed manifolds, the derivation of the locality principle in this model is not based on any index formula (and hence the model applies in situations where index formulas are yet to be obtained). This abstract model serves as a source of relative index formulas (and, under additional assumptions like symmetry conditions, of index formulas) in various specific cases. By way of example, we consider applications to the index of elliptic operators on closed manifolds, the index of elliptic boundary value problems, and the index of elliptic operators (pseudodifferential operators and Fourier integral operators) on manifolds with singularities. While in the first two cases one deals with known theorems or their generalizations, essentially new results are obtained in the third case. Hence, let us discuss elliptic theory on singular manifolds in a little more detail. More precisely, we speak only of manifolds with isolated singularities.

Apparently, the main feature distinguishing the theory from its 'smooth' counterpart is the fact that for the case of singular manifolds, elements of the Calkin algebra of the algebra of pseudodifferential operators are pairs (principal symbol, conormal trajectory). The second element of such a pair is a family of pseudodifferential operators with complex parameter $p$ on a smooth manifold without boundary, namely, on the base of the cone at the singular points. It turns out that the index of an elliptic operator is not determined by the principal symbol (as is the case on smooth manifolds) but depends also on the conormal symbol and may vary even if the conormal symbol is perturbed by a finitedimensional operator with smooth kernel. Consequently, along with finite-dimensional (topological) information, the index formula must necessarily contain infinite-dimensional (functional-analytic) in formation in the from of dependence on the conormal symbol. Thus, the main paradigm of index theory on smooth manifolds, stating that the index must be expressed in topological terms, is no longer adequate for singular manifolds. (A similar situation arises for boundary value problems with (nonlocal) conditions of AtiyahPatodi-Singer type, where infinite-dimensional information enters the expression for the index via the so-called eta invariant.) It is natural to ask for conditions that must be imposed on a 'good' index formula in this case. The simplest, most natural condition 
here is as follows: the infinite-dimensional (analytic) information in an index formula must be separated from the finite-dimensional (topological) information. In other words, index formulas should be sought in the form

$$
\text { ind } A=f\left([\sigma(A)], \sigma_{c}(A)\right) \text {, }
$$

where $[\sigma(A)]$ is the equivalence class of the principal symbol in the corresponding $K$ theory. Thus, the right-hand side of a 'good' index formula should be a homotopy invariant of the principal symbol. However, none of the numerous index formulas known in the literature satisfies this condition. This is by no means a mere occasion, for a formula of the type (0.2) is impossible in principle on the set of all elliptic $\Psi$ DO: the index of a $\Psi D O$ on a singular manifold is not uniquely determined by the equivalence class of its principal symbol and by the conormal symbol. Hence the problem of finding an index formula in the class of all elliptic $\Psi D O$ has little topological meaning, and one is forced to seek narrower classes of operators in which a formula of the type (0.2) is possible. One of such classes is the class of operators, satisfying the so-called symmetry condition. For such operators, one has formula (0.2) even in the stronger form

$$
\text { ind } A=f_{1}([\sigma(A)])+f_{2}\left(\sigma_{c}(A)\right)
$$

(that is, the index splits into the sum of homotopy invariant terms corresponding to components of the corresponding element of the Calkin algebra). Moreover, the second term can be expressed via rather convenient analytic invariants, namely, the multiplicities of singular points of the conormal symbol. The same multiplicities occur in the index formula for quantized canonical transformations (Fourier integral operators), which emphasizes their important role in elliptic theory on singular manifolds. The proof of formula (0.3) is based on the abstract locality principle for the relative index in conjunction with surgery.

The structure of the paper is clear from the table of contents. We only point out that the full version submitted for publication to Russian Mathematical Surveys contains a detailed review of the main facts of elliptic theory on manifolds with isolated singularities, intended to make the paper self-contained and available to wider readership. This part is omitted from the preprint. We also note that some parts of the survey were previously published as separate preprints by the authors (sometimes in a slightly different form). Nevertheless, they are included here to preserve the integrity of the presentation.

\section{The abstract locality principle}

\subsection{Bottleneck spaces}

Bottleneck spaces are a natural framework in which one can deal with surgeries and prove a rather general relative index theorem. They were introduced in [30, 26, 29, 31]. 
Consider the algebra $C^{\infty}([-1,1])$ of smooth functions $\varphi(t), t \in[-1,1]$, on the interval $[-1,1]$ with topology given by the standard system of seminorms

$$
\|\varphi\|_{k}=\sup _{t \in[-1,1]}\left|\varphi^{(k)}(t)\right|
$$

The multiplication in $C^{\infty}([-1,1])$ is defined pointwise. Obviously, $C^{\infty}([-1,1])$ is a unital topological algebra with unit 1 being the function identically equal to 1 for all $t \in[-1,1]$.

Definition 1.1. A bottleneck space is a separable Hilbert space $H$ equipped with the structure of a module over the commutative topological algebra $C^{\infty}([-1,1])$ (the action is continuous, and the unit function $\mathbf{1} \in C^{\infty}([-1,1])$ acts as the identity operator in $\left.H\right)$.

Example 1.2. Let $W$ be an arbitrary separable Hilbert space. Then the Hilbert space $H=L^{2}([-1,1], W)$ of equivalence classes of measurable functions

$$
f:[-1,1] \longrightarrow W
$$

with finite norm

$$
\|f\|_{H}=\left\{\int_{-1}^{1}\|f(t)\|_{W}^{2} d t\right\}^{1 / 2}
$$

is a bottleneck measure with respect to the action of $C^{\infty}([-1,1])$ given by the pointwise multiplication

$$
(\varphi f)(t)=\varphi(t) f(t), \quad \varphi \in C^{\infty}([-1,1]), \quad f \in H .
$$

Example 1.3. Let $M$ be a compact $C^{\infty}$ manifold without boundary, and let

$$
\chi: M \longrightarrow[-1,1]
$$

be a smooth mapping. Then each Sobolev space $H^{s}(M)$ bears a natural action of $C^{\infty}([-1,1])$ given by the formula

$$
(\varphi f)(x)=\varphi(\chi(x)) f(x), \quad x \in M,
$$

for any $\varphi \in C^{\infty}([-1,1])$ and $f \in H^{s}(M)$. This action makes $H^{s}(M)$ a bottleneck space.

Remark 1.4. In this example (and in more general cases, where $M$ need not be a compact $C^{\infty}$ manifold without boundary), the subset

$$
U=\overline{\chi^{-1}(-1,1)} \subset M,
$$

where the bar stands for the closure, will be referred to as the bottleneck. Figure 1.1 clarifies the term. Here $\chi=-1$ to the left and $\chi=+1$ to the right of the bottleneck $U$ (dashed). 


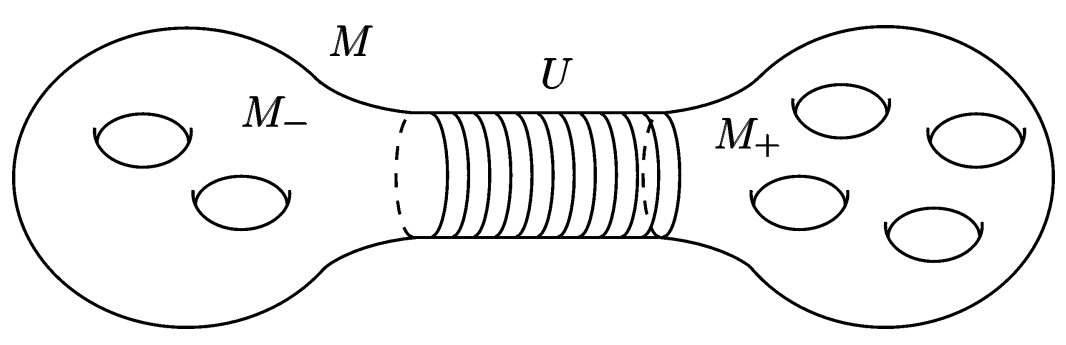

Figure 1.1: A bottleneck space.

Let $H$ be a bottleneck space. Then for each $h \in H$ one can naturally define the support of supp $h$ as a subset of $[-1,1]$. Moreover, supp $h$ coincides with the ordinary support of $h$ in example 1.2 and with the closure of the image of the ordinary support of $h$ under the mapping $\chi$ in example 1.3. The precise definition is as follows.

Definition 1.5. Let $H$ be a bottleneck space and $h \in H$ an arbitrary element. The support of $h$ is the closed set

$$
\operatorname{supp} h=\bigcap \varphi^{-1}(0) \subset[-1,1],
$$

where $\varphi^{-1}(0)$ is the preimage of the point 0 and the intersection is taken over all elements $\varphi \in C^{\infty}([-1,1])$ such that $\varphi h=0$.

The proof of the following assertion is trivial, and we omit it.

Proposition 1.6. The supports of elements of a bottleneck space $H$ have the following properties:

(i) $\operatorname{supp} h$ is a closed subset;

(ii) $\operatorname{supp} h=\varnothing \Leftrightarrow h=0$;

(iii) $\operatorname{supp}\left(h_{1}+h_{2}\right) \subset \operatorname{supp} h_{1} \cup \operatorname{supp} h_{2}$;

(iv) $\operatorname{supp}(\varphi h) \subset \operatorname{supp} \varphi \cap \operatorname{supp} h, \varphi \in C^{\infty}([-1,1])$;

(v) if $h_{n} \rightarrow h$ is a convergent sequence in $H$ (weakly or strongly), then

$$
\operatorname{supp} h \subset \bigcap_{k} \overline{\bigcup_{n \geq k} \operatorname{supp} h_{n}}
$$

where the bar stands for the closure.

Now let $F \subset[-1,1]$ be an arbitrary subset. Consider the subset

$$
\stackrel{\circ}{H}_{F}=\{h \in H \mid \operatorname{supp} h \subset F\}
$$

of a bottleneck space $H$. Obviously, this is a linear manifold (lineal) in $H$. 
Proposition 1.7. Let $F \subset[-1,1]$ be closed. Then so is $\stackrel{\circ}{H}_{F}$.

The proof readily follows from item (v) of Proposition 1.6.

For an arbitrary subset $F \subset[-1,1]$, we define $H_{F} \subset H$ as the closure of $\stackrel{\circ}{H}_{F}$.

Remark 1.8. In general, $H_{F} \neq H_{\bar{F}}$. In particular, in Example 1.3 one has

$$
H^{s}(M)_{(-1,1)}=\left\{u \in H^{s}(M) \mid \operatorname{supp} u \subset \overline{\chi^{-1}(-1,1)}\right\}
$$

(i.e., $H^{s}(M)_{(-1,1)}$ consists of functions supported in the bottleneck),

$$
H^{s}(M)_{[-1,1]}=H^{s}(M) .
$$

(Here $\operatorname{supp} u$ on the right-hand side of the first formula is the usual support of $u$.)

Now let $F_{1}, \ldots, F_{m} \subset[-1,1]$ be disjoint subsets. In general, it is not true that

$$
H_{F_{1} \cup \cdots \cup F_{m}}=H_{F_{1}} \oplus \cdots \oplus H_{F_{m}}
$$

(in particular, one usually has $H_{F} \oplus H_{[-1,1] \backslash F} \neq H$ ). But this is true if the closures of $F_{1}, \ldots, F_{m}$ are disjoint (in particular, if the $F_{j}$ themselves are closed).

Proposition 1.9. Let $F_{1}, \ldots, F_{m} \subset[-1,1]$ be subsets such that

$$
\bar{F}_{j} \cap \bar{F}_{k}=\varnothing \quad \text { for } \quad j \neq k .
$$

Then

$$
H_{F_{1} \cup \cdots \cup F_{m}}=H_{F_{1}} \oplus \cdots \oplus H_{F_{m}}
$$

(the sum is direct (1.2), but not necessarily orthogonal).

Propositions 1.6 and 1.9 show that supports of elements of bottleneck spaces have the same natural properties as usual supports of functions.

\section{$1.2 \quad$ Elliptic operators}

For operators in function spaces for which the Schwartz kernel theorem holds, there is an important notion of the support of the kernel, which is a closed subset of the direct product of the set where the functions are defined by itself. Although operators in bottleneck spaces cannot be described as integral operators in general, the notion of the support of an operator defined as a subset of the square $[-1,1] \times[-1,1]$ is meaningful and proves useful in studying various questions pertaining to the relative index. 
Definition 1.10. Let $A: H_{1} \longrightarrow H_{2}$ be a continuous linear operator in bottleneck spaces and $K \subset[-1,1] \times[-1,1]$ a closed subset. We say that the support of $A$ is contained in $K$ if

$$
\operatorname{supp} A h \subset K(\operatorname{supp} h)
$$

for every $h \in H$. In formula (1.3), $K$ is treated as a self-multimapping of the interval $[-1,1]$ :

$$
K x \stackrel{\text { def }}{=}\{y \in[-1,1] \mid(x, y) \in K\} .
$$

The intersection of all closed sets $K$ with property (1.3), is called the support of $A$ and denoted by supp $A$.

Let

$$
A: H_{1} \longrightarrow H_{2}, \quad B: H_{2} \longrightarrow H_{3}
$$

be operators in bottleneck spaces. Then the following composition theorem holds.

Proposition 1.11. One has

$$
\operatorname{supp}(B A) \subset \operatorname{supp} B \circ \operatorname{supp} A,
$$

where the right-hand side is understood as the composition of multimappings:

$$
\begin{aligned}
K_{2} \circ K_{1}=\left\{(x, z) \in[-1,1] \times[-1,1] \mid(x, y) \in K_{1},(y, z)\right. & \in K_{2} \\
& \quad \text { for some } y \in[-1,1]\} .
\end{aligned}
$$

In the theory of (pseudo)differential operators, the (pseudo)locality property plays and important role: the kernel is supported (for $\Psi D O$, modulo smooth functions) on the diagonal. An analog of this property proves useful in the relative index theorem for operators in bottleneck spaces. We state this analog in a form applicable to Fourier integral operators, which, despite not being pseudolocal, satisfy our wider definition in the cases of interest to us.

Let $\Delta \subset[-1,1] \times[-1,1]$ be the diagonal

$$
\Delta=\{(x, x) \mid x \in[-1,1]\},
$$

and let

$$
\Delta_{\varepsilon}=\{(x, y) \in[-1,1] \times[-1,1]|| x-y \mid<\varepsilon\}
$$

be the $\varepsilon$-neighborhood of $\Delta$.

Definition 1.12. A proper operator in bottleneck spaces $H_{1}$ and $H_{2}$ is a family of continuous linear operators

$$
A_{\delta}: H_{1} \longrightarrow H_{2}
$$

with parameter $\delta>0$ such that

(i) $A_{\delta}$ continuously depends on $\delta$ in the uniform operator topology;

(ii) for each $\varepsilon>0$ there is a $\delta_{0}>0$ such that

$$
\operatorname{supp} A_{\delta} \subset \Delta_{\varepsilon} \quad \text { for } \quad \delta<\delta_{0} \text {. }
$$


Remark 1.13. Condition (1.8) can be restated as follows: for $\delta<\delta_{0}$, one has

$$
\operatorname{supp} A_{\delta} h \subset U_{\varepsilon}(\operatorname{supp} h)
$$

for every $h \in H_{1}$, where $U_{\varepsilon}(F)$ is the $\varepsilon$-neighborhood of a set $F$.

Example 1.14. In the situation of Example 1.3, $\Psi D O$ in Sobolev spaces can naturally be viewed as proper operators (more precisely, included in the corresponding families) as follows. Let

$$
A: H^{s}(M) \longrightarrow H^{s-l}(M)
$$

be a $\Psi$ DO of order $l$ with kernel $A(x, y), x, y \in M$, on a smooth compact manifold $M$. Next, let $\varphi_{\delta}(x, y)$ be a smoothly depending on $\delta$ cutoff function on $M \times M$ equal to 1 in the $\delta$-neighborhood of the diagonal and vanishing outside the $2 \delta$-neighborhood. (We assume that $M$ is equipped with a Riemannian metric.) The operator $A_{\delta}$ with kernel $\varphi_{\delta}(x, y) A(x, y)$ is a $\Psi D O$ with the same symbol as $A$ and is equal to $A$ for sufficiently large $\delta$. One can readily see that the family $A_{\delta}$ satisfies the conditions of definition 1.12.

Remark 1.15. The product and the sum of proper operators (if they are well define) are proper operators; this readily follows from Definition 1.12, Proposition 1.11, and the triangle inequality. In particular, proper operators in a given bottleneck space $H$ form an algebra.

Now we can give the definition of elliptic operators in bottleneck spaces.

Definition 1.16. An elliptic operator in bottleneck spaces $H$ and $G$ is a proper operator

$$
D_{\delta}: H \longrightarrow G
$$

such that $D_{\delta}$ is Fredholm for each $\delta$ and has an almost inverse $D_{\delta}^{[-1]}$ such that the family $D_{\delta}^{[-1]}$ is also a proper operator.

Here, as usual, the almost inverse of a bounded operator $A$ is defined as an operator $A^{[-1]}$ such that the products $A A^{[-1]}$ and $A^{[-1]} A$ differ from the identity operators by compact operators in the corresponding spaces.

Example 1.17. In the situation of Example 1.3, an elliptic $\Psi D O A$ in Sobolev spaces on a smooth compact manifold $M$ can be treated as an elliptic operator in bottleneck spaces (i.e., included in an appropriate family). To this end, it suffices to apply the construction of Example 1.14 to the operator $A$ itself an to an arbitrary $\Psi D O A^{[-1]}$ with principal symbol being the inverse of that of $A$. Since the family $A_{\delta}$ is continuous and elliptic, it consists of representatives of the same stable homotopy class $[A] \in \operatorname{Ell}(M)$ of elliptic $\Psi \mathrm{DO}$ on $M$, and so the replacement of the operator by a family is valid in the framework of index theory. This justifies our terminology, which does not distinguish between an operator and the corresponding family. As a rule, we omit the parameter $\delta$ in the notation of a proper (in particular, elliptic) operator. 
Remark 1.18. For $X=[-1,1]$, the class of elliptic operators in bottleneck spaces is wider than the class of general elliptic operators introduced by Atiyah [5] and consisting of Fredholm operators $A: H \longrightarrow G$ acting in Hilbert $C(X)$-modules $H$ and $G$ (where $C(X)$ is the $C^{*}$-algebra of continuous functions on a compact set $X$ ) and commuting with the action of $C(X)$ modulo compact operators.

\subsection{The relative index theorem}

Most of the proofs pertaining to index formulas in this survey are carried out with the help of surgery, which is a method permitting one to cut and paste (parts of) manifolds together with operators defined there and keep track of the variation in the index under these operations. The index variation is governed by a statement which will be referred to as the relative index theorem. Here we state it in quite a general form, namely, in the framework of bottleneck spaces, following [25, 26].

First of all, we describe surgery itself in terms of bottleneck spaces. The corresponding notion in this context is modifications of bottleneck spaces, introduced in the following definition.

Let $H_{1}$ and $H_{2}$ be bottleneck spaces.

Definition 1.19. If for some $F \subset[-1,1]$ there is a given isomorphism (not necessarily isometric)

$$
j: H_{1}(F) \cong H_{2}(F),
$$

then we say that $H_{1}$ and $H_{2}$ coincide on $F$ (or are modifications of each other on $[-1,1] \backslash F)$. In this case, we write

$$
H_{1} \stackrel{F}{=} H_{2} \text { or } H_{1} \stackrel{[-1,1] \backslash F}{\longleftrightarrow} H_{2} .
$$

We point out that the specific form of the isomorphism is important here (rather than the existence of some isomorphism, which is always the case if $H_{1}(F)$ and $H_{2}(F)$ have the same dimension).

Example 1.20. Suppose that we modify a manifold $M$ (Fig. 1.1) by replacing $M_{-}$by another manifold $M_{-}^{\prime}$ with the same boundary $\partial M_{-}^{\prime}=\partial M_{-}$(cutting and pasting), thus obtaining a new manifold $M^{\prime}$ (a modification), shown in Fig. 1.2. Then we can equip $H^{s}\left(M^{\prime}\right)$ with the structure of a bottleneck space in the same way as above, with the help of a function $\chi^{\prime}$ given by the formula

$$
\chi^{\prime}(x)= \begin{cases}\chi(x), & x \in M_{+} \cup U, \\ -1, & x \in M_{-}^{\prime} .\end{cases}
$$




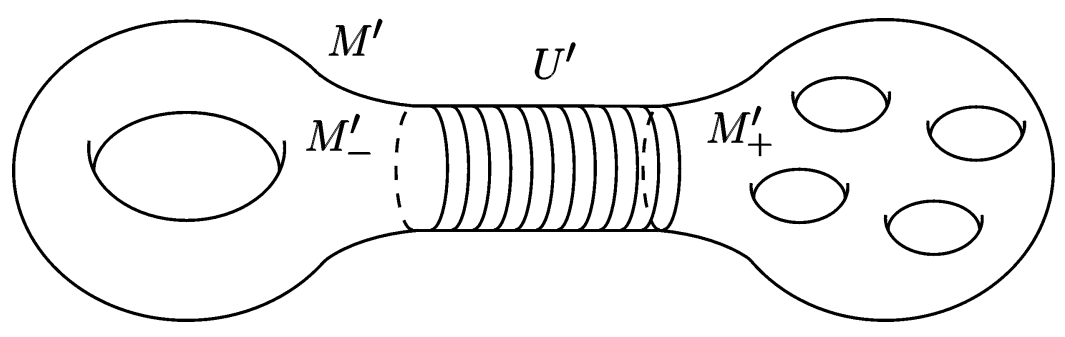

Figure 1.2: Surgery on a manifold $M$.

Then $H^{s}(M) \stackrel{(-1,1]}{=} H^{s}\left(M^{\prime}\right)$ or, equivalently, $H^{s}(M) \stackrel{-1}{\longleftrightarrow} H^{s}\left(M^{\prime}\right)$. (To simplify the notation, we write $H_{1} \stackrel{-1}{\longleftrightarrow} H_{2}$ and $H_{1} \stackrel{1}{\longleftrightarrow} H_{2}$ instead of $H_{1} \stackrel{\{-1\}}{\longleftrightarrow} H_{2}$ and $H_{1} \stackrel{\{1\}}{\longleftrightarrow} H_{2}$, respectively.)

Now we can extend the notion of modifications to operators.

Definition 1.21. Let $F \subset[-1,1]$ be an open subset, and let $H_{1} \stackrel{F}{=} H_{2}$ and $G_{1} \stackrel{F}{=} G_{2}$ be bottleneck spaces. We say that proper operators

$$
\begin{aligned}
& A_{1}: H_{1} \rightarrow G_{1}, \\
& A_{2}: H_{2} \rightarrow G_{2}
\end{aligned}
$$

coincide on $F$ if for each compact subset $K \subset F$ the following condition is satisfied: there is a number $\delta_{0}=\delta_{0}(K)>0$ such that

$$
A_{1 \delta} h=A_{2 \delta} h
$$

whenever $\delta<\delta_{0}$ and supp $h \subset K$.

We note that (1.9) is well defined, since $h \in H_{1}(K) \cong H_{2}(K)$ and for small $\delta$ one has $A_{1 \delta} h, A_{2 \delta} h \in G_{1}(F) \cong G_{2}(F)$; the latter inclusion follows from the fact that $F$ is open.

In the conditions of Definition 1.21, we say that $A_{1}$ is obtained from $A_{2}$ by a modification on $[-1,1] \backslash F$ (or $A_{1}$ coincides with $A_{2}$ on $F$ ) and write $A_{1} \stackrel{F}{=} A_{2}$ or $A_{1} \stackrel{[-1,1] \backslash F}{\longleftrightarrow} A_{2}$.

We return to the example of the modification given by the transition $M \rightarrow M^{\prime}$ (see Figs. 1.1 and 1.2). If on $M$ and $M^{\prime}$ there are given differential operators $D$ and $D^{\prime}$ coinciding on $U \cup M_{+}$, then $D \stackrel{-1}{\longleftrightarrow} D^{\prime}$ (families independent of $\delta$ ). More generally, let $D$ and $D^{\prime}$ be $\Psi D O$ on $M$ and $M^{\prime}$, respectively, with principal symbols satisfying the condition

$$
\sigma(D)=\sigma\left(D^{\prime}\right) \quad \text { over } \quad U \cup M_{+} .
$$

Then, using homotopies adding lower-order operators to $D$ and $D^{\prime}$, one can include these operators in families coinciding on $(-1,1]$ in the sense of Definition 1.21. By abuse of notation, in this case we also write $D \stackrel{-1}{\longleftrightarrow} D^{\prime}$. 
In the following, we deal with diagrams of modifications, more precisely, squares of the form

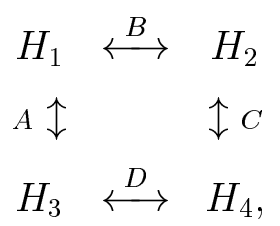

where the $H_{i}$ are bottleneck spaces and $A, B, C, D \subset[-1,1]$. This square is said to commute if the diagram

$$
\begin{aligned}
& H_{1}(F) \approx H_{2}(F) \\
& \text { थ } 2 \quad F=[-1,1] \backslash\{A \cup B \cup C \cup D\} \text {, } \\
& H_{3}(F) \approx H_{4}(F) \text {, }
\end{aligned}
$$

of isomorphisms commutes, where the arrows are the restrictions to the relevant subspaces of the corresponding isomorphisms occurring in Definition 1.19. A similar square of modifications for operators is said to commute if the underlying square of modifications of bottleneck spaces commutes.

Now we are in a position to state the main theorem of this section.

Theorem 1.22 ([25, 26]). Suppose that the following commutative diagram of modifications of elliptic operators in bottleneck spaces holds:

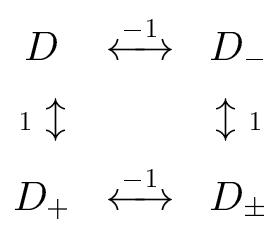

Then

$$
\operatorname{ind}(D)-\operatorname{ind}\left(D_{-}\right)=\operatorname{ind}\left(D_{+}\right)-\operatorname{ind}\left(D_{ \pm}\right) .
$$

A detailed proof of this theorem (which however occupies less than two pages) can be found in [25], and we omit it. Note only that this proof is based on the following two lemmas about proper operators in bottleneck spaces, which are of interest in themselves. (These lemmas and their proofs can also be found in [25].

Lemma 1.23. Let $A_{1} \stackrel{F}{=} A_{2}$ be elliptic operators in bottleneck spaces. Then $A_{1}^{[-1]} \stackrel{F}{=}$ $A_{2}^{[-1]}+K$, where $K$ is a proper compact operator. In particular, for $A_{1}=A_{2}=A$ we find that two arbitrary almost inverses of $A$ differ by a proper compact operator.

Lemma 1.24. Suppose that $[-1,1]=\bigcup_{j} F_{j}$ is an open cover and $A_{1} \stackrel{F_{j}}{=} A_{2}$ for all $j$, where $A_{1}$ and $A_{2}$ are proper operators. Then $A_{1 \delta}=A_{2 \delta}$ for sufficiently small $\delta$.

In the next sections, we show how the general relative index theorem 1.22 almost trivially implies various relative index theorems in specific cases. 


\section{Localization in index theory on smooth manifolds}

\subsection{Surgery on compact manifolds}

\subsubsection{The general construction}

We use only the simplest surgery defined as follows (see [26]). Let $M$ be a compact smooth manifold without boundary divided into two parts $M_{-}$and $M_{+}$by a smooth hypersurface $S$ (Fig. 1.1), and let $D$ be an elliptic differential operator acting between spaces of sections of some vector bundles $E$ and $F$ over $M$. We cut off $M_{-}$along $S$ and attach another manifold $M_{-}^{\prime}$ to $M_{+}$such that $M_{-}^{\prime} \bigcup_{S} M_{+}$is again a smooth compact manifold $M^{\prime}$ without boundary (Fig. 1.2). Needless to say, to define a smooth structure on the manifold obtained by gluing, we must choose some direct product structure in a collar neighborhood of $S$ in each of the manifolds considered here. Next, we extend the bundles $\left.E\right|_{M_{+}}$and $\left.F\right|_{M_{+}}$to bundles over the entire $M^{\prime}$ and the operator $\left.D\right|_{M_{+}}$to an elliptic operator $D^{\prime}$ on the entire $M^{\prime}$ in spaces of sections of these new bundles. (We assume that this is possible.) The difference

$$
\operatorname{ind}\left(D^{\prime}, D\right)=\operatorname{ind} D^{\prime}-\text { ind } D
$$

of indices of $D^{\prime}$ and $D$ is called the relative index of these operators. Let us perform the same cut-and-paste operation on the right half of the manifold replacing $M_{+}$by some other part $\widetilde{M}_{+}$. We obtain new manifolds $\widetilde{M}$ and $\widetilde{M^{\prime}}$. Carrying out the corresponding operations on bundles and operators, we arrive at new operators $\widetilde{D}$ and $\widetilde{D}^{\prime}$, which still coincide on the right half of the manifolds, that is, on $\widetilde{M}_{+}$in this case, while on the left half they coincide with $D$ and $D^{\prime}$, respectively. We readily see that by homotopying all four operators in the class of elliptic operators, one can ensure that they all coincide with one another in $U$. The general relative index theorem 1.22 implies that

$$
\operatorname{ind}\left(D^{\prime}, D\right)=\operatorname{ind}\left(\widetilde{D}^{\prime}, \widetilde{D}\right) .
$$

(However, Eq. (2.2) for $C^{\infty}$ manifolds trivially follows from the so-called local index formula (e.g., see [15]): the index of an elliptic operator $D$ on a closed manifold $M$ is given by the expression

$$
\text { ind } D=\int_{M} \alpha(x) \text {, }
$$

where the "local density" $\alpha(x)$ at a point $x \in M$ depends only on the principal symbol $\sigma(D)$ and its derivatives in the fiber over $x$.)

We shall apply this construction in the next subsection.

Remark 2.1. If one rejects the smoothness condition and only requires that $M$ be a Lipschitz manifold, then the above construction in conjunction with Theorem 1.22 gives a theorem from Teleman's paper [43]. 


\subsubsection{The Booß-Wojciechowski theorem}

In the present subsection we study how the index of an operator that is "locally" an elliptic $\psi \mathrm{DO}$ on a manifold is changed if one performs surgery on the manifold where the operator is defined and some associated surgery on the bundles. The index increment formula can naturally be treated as a relative index formula. The informal motivation is as follows: the symbol before and after the surgery is essentially the same; we deal with different realizations of the same symbol, which depend on the way in which the manifold was glued from pieces. This theorem (for the case of smooth manifolds) is contained in [8, Chap. 25]. The construction is of certain interest, even though it is a special case of the construction from the preceding subsection.

Let $M$ be an orientable manifold (possibly, with boundary and/or singularities), and let $S \subset M$ be an embedded smooth compact two-sided submanifold of codimension 1 contained in the smooth "interior" part of $M$. Next, let $U$ be a collar neighborhood of $S$ contained in the smooth part of $M$. We choose and fix some trivialization $U=(-1,1) \times S$ of this neighborhood and use the coordinates $(t, s), t \in(0,1), s \in S$, there. Let $g: S \rightarrow S$ be a given diffeomorphism. We perform the following operation: we cut $M$ along $S$ and glue together again, identifying each point $(-0, s)$ on the left coast of the cut with the corresponding point $(+0, g(s))$ on the right coast. The resultant smooth manifold (the smooth structure is well defined, since we have chosen and fixed the trivialization) will be denoted by $M_{g}$ and called the surgery of $M$ via $g$.

Let $E$ be a vector bundle over $M$. Suppose that we are given an isomorphism of vector bundles

$$
\mu:\left.E\right|_{S} \rightarrow g^{*}\left(\left.E\right|_{S}\right) .
$$

Then over $M_{g}$ there is a naturally defined vector bundle $E_{g, \mu}$ (by attaching along $S$ with the help of $\mu$ ), which will be called the surgery of $E$ via the pair $(g, \mu)$.

Now let $E, F$ be two vector bundles over $M$, and let

$$
a: \pi^{*} E \rightarrow \pi^{*} F,
$$

where $\pi: T_{0}^{*} M \rightarrow T_{0}^{*} M$ is the natural projection, be an elliptic symbol of some order $m$. By choosing the representation

$$
\left.E\right|_{U}=\tilde{\pi}^{-1}\left(\left.E\right|_{S}\right),\left.\quad F\right|_{U}=\tilde{\pi}^{-1}\left(\left.F\right|_{S}\right)
$$

of the bundles $E, F$ over $U=[0,1] \times S$, where $\widetilde{\pi}:[0,1] \times S \rightarrow S$ is the natural projection, and by passing to a homotopic symbol if necessary, we can assume that $a$ is independent of the coordinate $t$ in a sufficiently small neighborhood of $S$ (that is, $a \equiv a_{0}$ in that neighborhood). Consider the mapping (denoted by the same letter)

$$
\left.a_{0} \stackrel{\text { def }}{=} a\right|_{\pi^{-1} S}:\left.\left.\pi^{*} E\right|_{\pi^{-1} S} \longrightarrow \pi^{*} F\right|_{\pi^{-1} S} .
$$

With regard to the trivialization chosen, this mapping can be rewritten in the form

$$
a_{0}(p, s, \xi): E_{s} \longrightarrow F_{s}, \quad p^{2}+|\xi|^{2} \neq 0, \quad s \in S,
$$


where $p$ is the dual variable of $t$ and $\xi$ is a point in the fiber of $T^{*} S$ over $s$.

Suppose that a surgery $g$ of $M$ and associated surgeries $\mu_{E}$ and $\mu_{F}$ of the bundles $E$ and $F$ are given. If the diagram

$$
\begin{array}{ccc}
E_{s} \quad & a_{0}(p, s, \xi) & F_{s} \\
\downarrow \mu_{E}(s) & & \downarrow \mu_{F}(s) \\
E_{g(s)} \longrightarrow a_{0}\left(p, g(s),{ }^{t} g_{s}(s)^{-1} \xi\right) & & F_{g(s)},
\end{array}
$$

where ${ }^{t} g_{s}(s)$ is the transposed Jacobi matrix of the mapping $g$ at the point $s$, commutes, then the surgery takes the original symbol $a$ to a new smooth symbol $\widetilde{a}$ on the cotangent bundle $T_{0}^{*} M_{g}$. (The smoothness of the newly obtained symbol is guaranteed by the independence of $a$ on the coordinate $t$ in a neighborhood of $S$.) We intend to find out how the surgery affects the index of the corresponding $\Psi D O$ (or a $\Psi D O$ on a singular manifold, or a boundary value problem...). Since actually this index increment (the relative index) depends only on the surgery on $S$, we use the results of the preceding section to pass to the corresponding "local" model and simultaneously get rid of the necessity to state any explicit conditions on the boundary or in the nonsmooth part of the manifold.

Thus, let $A$ and $\widetilde{A}$ be operators with principal symbols $a$ and $\widetilde{a}$ on the manifolds $M$ and $M_{g}$, respectively, obtained from each other by the above-mentioned surgery. The problem is to find the relative index ind $\widetilde{A}-$ ind $A$.

It follows from Theorem 1.22 that the relative index is independent of the structure of the operators in question outside a small neighborhood of $S$. Hence we can use the simplest model for the computations. Namely, consider the manifold $M=S \times S^{1}$ and the elliptic pseudodifferential operator

$$
A_{0}: H^{s}(M, E) \rightarrow H^{s-m}(M, F)
$$

(the bundles $E$ and $F$ are lifted to $M$ with the help of the natural projection $M=$ $S \times S^{1} \rightarrow S$ ) with principal symbol $a_{0}$ independent of $\varphi \in S^{1}$. Next, let $M_{g}$ be the surgery of $M$ with the help of $g$, let $E_{g, \mu_{E}}$ and $E_{g, \mu_{F}}$ be the associated surgeries of the bundles $E$ and $F$, and let

$$
\widetilde{A}_{0}: H^{s}\left(M_{g}, E_{g, \mu_{E}}\right) \rightarrow H^{s-m}\left(M_{g}, E_{g, \mu_{F}}\right)
$$

be the new elliptic pseudodifferential operator with principal symbol $\widetilde{a}_{0}$ coinciding with $A_{0}$ outside a neighborhood of the set $S$, where the surgery is done.

The operators $A_{0}$ and $\widetilde{A}_{0}$ are elliptic operators on compact manifolds, and their index can be calculated by the Atiyah-Singer theorem. The index of $A_{0}$ is zero, since its symbol is independent of $\varphi \in S^{1}$. Hence in this model only one term in the expression for the relative index is nontrivial:

$$
\operatorname{ind} \widetilde{A}-\operatorname{ind} A=\operatorname{ind} \widetilde{A}_{0} \text {. }
$$

Let us state the assertion that we have just proved in the from of a theorem. 
Theorem 2.2. Let $S$ be a smooth compact manifold without boundary, and let $H$ and $G$ be bottleneck spaces coinciding in a neighborhood of zero with the spaces $H^{s}(C, E)$ and $H^{s-m}(C, F)$, respectively, where $E$ and $F$ are vector bundles over the manifold $C=$ $(-1,1) \times S$. (The structure of bottleneck spaces on $H^{s}(C, E)$ and $H^{s-m}(C, F)$ is defined via the mapping $\chi: C \rightarrow[-1,1], \quad(t, s) \mapsto t$.$) Next, let$

$$
A: H \longrightarrow G
$$

be an elliptic operator coinciding in a neighborhood of zero with an elliptic pseudodifferential operator with principal symbol a of order $m$ on $T_{0}^{*} C$. Let b be the restriction (2.4) of the symbol a to $\pi^{-1} S$. Suppose that we are given some surgery of $C$ via a diffeomorphism $g: S \rightarrow S$ and associated surgeries $\mu_{E}$ and $\mu_{F}$ of the bundles $E$ and $F$, and moreover, the diagram (2.5) commutes. Let $\widetilde{A}$ be the corresponding surgery of the operator $A$. Then the relative index of the surgery is given by the formula

$$
\text { ind } \widetilde{A}-\text { ind } A=\text { ind } \widetilde{A}_{0} \text {, }
$$

where $\widetilde{A}_{0}$ is the operator with symbol $\widetilde{a}_{0}$ on $T_{0}^{*}\left(S \times S^{1}\right)_{g}$ obtained by surgery from the homomorphism $a_{0}$ lifted to $T_{0}^{*}\left(S \times S^{1}\right)$ with the help of the natural projection along $S^{1}$.

\subsection{Surgery on noncompact manifolds}

The locality principle in index theory on non-compact manifolds was apparently obtained for the first time (for the special case of Dirac operators on noncompact Riemannian manifolds) by Gromov and Lawson [16], who obtained the corresponding relative index theorem. Later Anghel [3] generalized their result to arbitrary self-adjoint elliptic first-order operators on a complete Riemannian manifold. In fact, these relative index theorems are a straightforward consequence of the general results stated earlier in this paper. In this subsection, we briefly describe the result due to Gromov and Lawson.

\subsubsection{The Gromov-Lawson theorem}

Let $X_{0}$ and $X_{1}$ be complete even-dimensional Riemannian manifolds, and let $D_{0}$ and $D_{1}$ be generalized Dirac operators on $X_{0}$ and $X_{1}$, respectively, acting on sections of vector bundles $S_{1}$ and $S_{2}$. We say that $D_{0}$ and $D_{1}$ coincide at infinity if there exist compact subsets $K_{0} \subset X_{0}$ and $K_{1} \subset X_{1}$, an isometry

$$
F:\left(X_{0} \backslash K_{0}\right) \stackrel{\approx}{\rightarrow}\left(X_{1} \backslash K_{1}\right),
$$

and an isometry

$$
\widetilde{F}:\left.\left.S_{0}\right|_{X_{0} \backslash K_{0}} \rightarrow S_{1}\right|_{X_{1} \backslash K_{1}}
$$

of vector bundles such that

$$
D_{1}=\widetilde{F} \circ D_{0} \circ \widetilde{F}^{-1} \quad \text { on } \quad X_{1} \backslash K_{1} .
$$


To simplify the notation, we identify $X_{0} \backslash K_{0}$ with $X_{1} \backslash K_{1}$ and write

$$
D_{0}=D_{1} \quad \text { on } \quad \Omega=X_{0} \backslash K_{0} \cong X_{1} \backslash K_{1} .
$$

In this situation we can define the topological relative index $\operatorname{ind}_{t}\left(D_{1}^{+}, D_{0}^{+}\right)$of the operators

$$
D_{1}^{+}: \Gamma\left(S_{1}^{+}\right) \rightarrow \Gamma\left(S_{1}^{-}\right) \quad \text { and } \quad D_{0}^{+}: \Gamma\left(S_{0}^{+}\right) \rightarrow \Gamma\left(S_{0}^{-}\right)
$$

as follows. If $X_{0}$ and $X_{1}$ are compact, then we simply set

$$
\operatorname{ind}_{t}\left(D_{1}^{+}, D_{0}^{+}\right)=\operatorname{index}\left(D_{1}^{+}\right)-\operatorname{index}\left(D_{0}^{+}\right) .
$$

(The expression on the right-hand side is simply the difference of the usual analytical=topological indices of the operators $D_{1}^{+}$and $D_{0}^{+}$, expressed, say by the Atiyah-Singer formula). If $X_{0}$ (and hence $X_{1}$ ) is noncompact, then we use the following procedure. We cut the manifolds $X_{0}$ and $X_{1}$ along some compact hypersurface $H \subset \Omega$ and compactify them by attaching some compact manifold with boundary $H$. The operators $D_{0}^{+}$and $D_{1}^{+}$ can be extended to elliptic operators $\widetilde{D}_{0}^{+}$and $\widetilde{D}_{1}^{+}$on the compact manifolds thus obtained. Now we set

$$
\operatorname{ind}_{t}\left(D_{1}^{+}, D_{0}^{+}\right)=\operatorname{ind}\left(\widetilde{D}_{1}^{+}\right)-\operatorname{ind}\left(\widetilde{D}_{0}^{+}\right) .
$$

Using the formula

$$
\text { index }(A)=\operatorname{Tr}(1-R A)-\operatorname{Tr}(1-A R)
$$

for the index of an elliptic operator $A$ (here $R$ is a parametrix of $A$ ), the localization of kernels of pseudodifferential operators in a neighborhood of the diagonal, and a partition of unity, Gromov and Lawson proved that the right-hand side of (2.6) is independent of the arbitrariness in the above construction.

Next, let the operators $D_{0}^{+}$and $D_{1}^{+}$be positive at infinity (the precise definition is given in [16]; roughly speaking, this condition means that the free terms in the operators $\left(D_{0}^{+}\right)^{*} D_{0}$ and $\left(D_{1}^{+}\right)^{*} D_{1}$ expressed via covariant derivatives are positive). Then the operators $D_{0}^{+}$and $D_{1}^{+}$are Fredholm, and one can define the analytical relative index

$$
\operatorname{ind}_{a}\left(D_{1}^{+}, D_{0}^{+}\right)=\operatorname{ind}_{a}\left(D_{1}^{+}\right)-\operatorname{ind}_{a}\left(D_{0}^{+}\right) .
$$

The Gromov-Lawson relative index theorem states that the topological and analytical relative indices coincide:

$$
\operatorname{ind}_{a}\left(D_{1}^{+}, D_{0}^{+}\right)=\operatorname{ind}_{t}\left(D_{1}^{+}, D_{0}^{+}\right) .
$$

In [16] one can also find a more general theorem pertaining to the case in which the operators $D_{0}$ and $D_{1}$ coincide only on some of the "ends" of $X_{0}$ and $X_{1}$ at infinity. In this case, one again has a formula like (2.8), where the right-hand side is no longer the "topological relative index," but it is rather the analytical index of some elliptic Fredholm operator on a (generally speaking, noncompact) manifold obtained from $X_{0}$ and $X_{1}$ by 
cutting away the "common" ends along some hypersurface followed by gluing along that hypersurface. The proof uses the same technique.

We can conclude (as is easily seen from the second theorem) that the topological index actually has nothing to do with the Gromov-Lawson relative index theorem: this theorem states the equality of the analytical relative indices for two pairs of operators obtained from each other by simultaneous surgery on a part of the manifold where they coincide; the topological index occurs in the answer only if the newly obtained operators fall within the scope of the Atiyah-Singer theorem. (On the other hand, naturally, the applications of theorems of that type are just related to transforming the original operators to new operators such that the Atiyah-Singer theorem or any other theorem expressing the index in topological terms can be used.) As to the equality of analytic relative indices, it directly falls within the scope of Theorem 1.22.

\section{Localization for boundary value problems}

In this section, we describe some applications of the locality principle for the relative index to the theory of boundary value problems for elliptic differential operators.

\subsection{Some notation}

Let $X$ be a smooth compact $n$-dimensional manifold with boundary $\partial X=Y$ that is a smooth closed manifold of dimensional $n-1$. We choose and fix a representation of some collar neighborhood $U$ of the boundary in the form of a direct product

$$
U \simeq Y \times[0,1)
$$

where $Y$ is taken to $Y \times\{0\}$ by the identity mapping. The coordinate on $[0,1)$ will be denoted by $t$, an the local coordinates on the boundary by $y=\left(y_{1}, \ldots, y_{n-1}\right)$, so that local coordinates on $X$ in $U$ have the form

$$
x=\left(x_{1}, \ldots, x_{n}\right)=(y, t) .
$$

If $E$ is a vector bundle over $X$, then the restriction $\left.E\right|_{U}$ is isomorphic to the lift to $U$ of the restriction $\left.E\right|_{Y}$ of the same bundle to the boundary:

$$
\left.E_{U} \simeq \pi_{U}^{*} E\right|_{Y}
$$

where $\pi_{U}: U \rightarrow Y$ is the projection naturally associated with the representation (3.1)

Now let

$$
\widehat{D}: C^{\infty}\left(X, E_{1}\right) \rightarrow C^{\infty}\left(X, E_{2}\right)
$$

be an elliptic differential operator of order $m$ on $X$ acting in sections of finite-dimensional vector bundles $E_{1}$ and $E_{2}$. Then, using the trivialization (3.1) and the associated representations (3.2) of $E_{1}$ and $E_{2}$ over $U$ as the lifts of $\left.E_{1}\right|_{Y}$ and $\left.E_{2}\right|_{Y}$, we can represent the 
operator (3.3) in $U$ in the form

$$
\widehat{D}=\sum_{j=0}^{m} \widehat{D}_{j}(t)\left(-i \frac{\partial}{\partial t}\right)^{j}
$$

where

$$
\widehat{D}_{j}(t): C^{\infty}\left(Y,\left.E_{1}\right|_{Y}\right) \rightarrow C^{\infty}\left(Y,\left.E_{2}\right|_{Y}\right)
$$

is a differential operator of order $m-j$ in sections of bundles over $Y$, depending on the parameter $t$. Next, the coefficient $\widehat{D}_{m}(t)$ is a differential operator of order 0 , i.e., a bundle homomorphism, and since $\widehat{D}$ is elliptic, this coefficient is a bundle isomorphism. Dividing the operator $\widehat{D}$ in $U$ by this coefficient on the left, we can assume without loss of generality that the bundles $\left.E_{1}\right|_{Y}$ and $\left.E_{2}\right|_{Y}$ coincide and the coefficient itself is the identity operator.

The operator family

$$
\mathfrak{D}(p)=\sum_{j=0}^{m} \widehat{D}_{j}(0) p^{j}: H^{s}(Y) \rightarrow H^{s-m}(Y)
$$

acting in Sobolev spaces ${ }^{1}$ on $Y$ and obtained from the representation (3.4) by freezing the coefficients at the boundary $t=0$ and by replacing the operator $-i \partial / \partial t$ with the variable $p$ will be called the conormal symbol of the operator $\widehat{D}$.

If $u \in H^{s}(X)$ is an element of a Sobolev space on $X$, then for $s>m-1 / 2$ by trace theorems we have a well-defined jet of order $m-1$ of $u$ on $Y$. With regard to the identifications (3.1) and (3.2), it can be rewritten in the form

$$
j_{X}^{m-1} u=\left(\left.u\right|_{t=0},\left.\frac{\partial u}{\partial t}\right|_{t=0}, \ldots,\left.\frac{\partial^{m-1} u}{\partial t^{m-1}}\right|_{t=o}\right) \in H^{s-1 / 2}(Y) \oplus \cdots \oplus H^{s-m+1 / 2}(Y) .
$$

Boundary value problems for $\widehat{D}$ are stated in terms of the boundary jet (3.6) of $u$, to which one applies some differential or pseudodifferential operators. Since for $m>1$ the space on the right-hand side in (3.6), which for brevity will be denoted by

$$
\mathcal{H}_{m}^{s-1 / 2}(Y)=\bigoplus_{k=0}^{m-1} H^{s-k-1 / 2}(Y)
$$

is a direct sum of Sobolev spaces of distinct orders, the orders of $\Psi D O$ in such spaces must be understood in the sense of Douglis-Nirenberg.

\footnotetext{
${ }^{1}$ In what follows, we usually omit the bundles in the notation of Sobolev spaces.
} 


\subsection{General boundary value problem}

General boundary value problems, which include classical boundary value problems as well as nonlocal problems of the Atiyah-Patodi-Singer type (in particular, nonhomogeneous) were introduced in [39]. Let $\widehat{D}$ be an elliptic differential operator (3.3) on a manifold $X$. A general boundary value problem for $\widehat{D}$ is a problem of the form

$$
\left\{\begin{aligned}
\widehat{D} u & =f \in H^{s-m}(X), \\
\widehat{B} j_{Y}^{m-1} u & =g \in \mathcal{L},
\end{aligned}\right.
$$

where $s>m-1 / 2$, the element $u \in H^{s}(X)$ is to be found, $\mathcal{L}$ is a Hilbert space, and $\widehat{B}$ is a continuous linear operator in the spaces

$$
\widehat{B}: \mathcal{H}_{m}^{s-1 / 2}(Y) \rightarrow \mathcal{L} .
$$

Ordinary boundary value problems are the special case in which $\mathcal{L}$ is a Sobolev space of sections of some vector bundle over the boundary and $B$ is a (pseudo)differential operator. If $\widehat{D}$ is the Dirac operator on an even-dimensional manifold $X, \mathcal{L}$ is the positive spectral subspace of the tangential operator, and $B$ is the orthogonal projection on $\mathcal{L}$, then we arrive at the Atiyah-Patodi-Singer problem [6], more precisely, a more general problem in which the nonlocal boundary data may be nonzero.

As shown by these examples, of main interest is the case in which $\mathcal{L}$ is not an abstract Hilbert space but rather a subspace of some Sobolev space on the boundary ${ }^{2}$ and $B$ is a $\Psi D O$. More precisely, we shall consider only subspaces that are ranges of pseudodifferential projections. If $\widehat{P}$ is a pseudodifferential projection on some subspace $\widehat{L}$ of a Sobolev space of sections of some vector bundle $F$ over $Y$, then the principal symbol $P=\sigma(\widehat{P})$ is a projection on a subbundle $L \subset \pi^{*} F$ over $T_{0}^{*} Y$, where $\pi: T_{0}^{*} Y \rightarrow Y$ is the natural projection. The subbundle $L$ is called the principal symbol of $\widehat{L}$. The pseudodifferential version of the general boundary value problem (3.8) for an unknown function $u \in H^{s}\left(X, E_{1}\right)$ has the form

$$
\left\{\begin{aligned}
\widehat{D} u & =f \in H^{s-m}\left(X, E_{2}\right), \\
\widehat{B} j_{Y}^{m-1} u & =g \in \widehat{P} H(Y, F),
\end{aligned}\right.
$$

where $H(Y, F)$ is a Sobolev space of sections of a bundle $F$ over the boundary (we intentionally omit the index on this space, since it can be a usual Sobolev space or a space of the form $\left.\mathcal{H}_{m}^{s}(Y)\right)$ and $\widehat{B}: \mathcal{H}_{m}^{s-1 / 2} \rightarrow H(Y, F)$ - is a $\Psi$ DO such that $R(\widehat{B}) \subseteq R(\widehat{P})$. (The last inclusion necessarily implies that $R(B) \subseteq R(P)=L$.)

Of general boundary value problems (3.8), we single out problems that are a straightforward (nonhomogeneous) analog of the Atiyah-Patodi-Singer problem. Namely, let an operator $\widehat{D}$ of order $m$ be given. On the basis of the conormal symbol (3.5) of $\widehat{D}$, we shall construct a pseudodifferential projection

$$
\widehat{P}_{+}: \mathcal{H}_{m}^{s}(Y) \rightarrow \mathcal{H}_{m}^{s}(Y)
$$

\footnotetext{
${ }^{2}$ In particular, the entire Sobolev space.
} 
in the Cauchy data space (3.7). The construction is as follows (see [27]). On the basis of the operator family (3.5), we construct the matrix operator

$$
\mathfrak{A}=\left(\begin{array}{ccccc}
0 & 1 & 0 & \ldots & 0 \\
0 & 0 & 1 & \ldots & 0 \\
0 & 0 & 0 & \ldots & 1 \\
-\widehat{D}_{0} & -\widehat{D}_{1} & -\widehat{D}_{2} & \ldots & -\widehat{D}_{n-1}
\end{array}\right): \mathcal{H}_{m}^{s}(Y) \rightarrow \mathcal{H}_{m}^{s-1}(Y)
$$

where $\widehat{D}_{j} \stackrel{\text { def }}{=} \widehat{D}_{j}(0)$, in the Cauchy data space. The operator $p-\mathfrak{A}, p \in \mathbb{C}$, is invertible if and only if so is the operator $\mathfrak{D}(p)$, and moreover,

$$
(p-\mathfrak{A})^{-1}=\mathfrak{D}(p)^{-1} \mathfrak{Q}(p),
$$

where the entries $\widehat{q}_{j k}(p), j, k=0, \ldots, m-1$ of the matrix $\mathfrak{Q}(p)$ are differential operators polynomially depending on $p$ whose total order (with regard to the parameter, to which we assign the unit weight) does not exceed

$$
\operatorname{ord} \widehat{q}_{j k}(p) \leq m-1+j-k \text {. }
$$

Since the operator $\widehat{D}$ is elliptic, it follows that the polynomial family $\mathfrak{D}(p)$ is AgranovichVishik elliptic with parameter $p[1]$ in the double sector

$$
\Lambda_{\varepsilon}=\{|\arg p|<\varepsilon\} \cup\{|\pi-\arg p|<\varepsilon\}
$$

on the complex $p$-plane for some $\varepsilon>0$ and elliptic in the usual sense for all $p \in \mathbb{C}$. It follows that the family $\mathfrak{D}(p)$ is finitely meromorphically invertible in the entire complex plane and the sector $\Lambda_{\varepsilon}$ contains only finitely many poles of the operator function $\mathfrak{D}^{-1}(p)$. Thus, the operator $\mathfrak{D}(p)$ (and hence $p-\mathfrak{A}$ ) is invertible on the line $\operatorname{Im} p=\delta$ for all sufficiently small $\delta>0$.

We set

$$
\widehat{P}_{+}=-\frac{\widehat{\mathfrak{A}}}{2 \pi i} \int_{-\infty+i \delta}^{+\infty+i \delta}(p-\mathfrak{A})^{-1} \frac{d p}{p},
$$

where $\delta>0$ is sufficiently small. This integral specifies a well-defined continuous projection

$$
\widehat{P}_{+}: \mathcal{H}_{m}^{s}(Y) \rightarrow \mathcal{H}_{m}^{s}(Y)
$$

in the Cauchy data space (e.g., see [27]). This projection corresponds to the spectral points of the operator $\mathfrak{A}$ in the upper half-plane. If $\widehat{D}$ is the Dirac operator, then the projection $\widehat{P}_{+}$thus introduced coincides with the Atiyah-Patodi-Singer spectral projection. In the following, we also set

$$
\widehat{P}_{-} \stackrel{\text { def }}{=} 1-\widehat{P}_{+} \text {. }
$$


The spectral boundary value problem is problem (3.8) of the special form

$$
\left\{\begin{array}{l}
\widehat{D} u=f \in \mathcal{H}^{s-u}(X) \\
\widehat{P}_{+} j_{Y}^{m-1} u=g \in \widehat{P}_{+} \mathcal{H}_{m}^{s-1 / 2}(Y) .
\end{array}\right.
$$

Problem (3.15), which will be denoted by $\left(\widehat{D}, \widehat{P}_{+}\right)$, is always Fredholm. The index of a general Fredholm boundary value problem $(3.8)$, which will be denoted by $(\widehat{D}, \widehat{B})$, is expressed by the formula

$$
\operatorname{ind}(\widehat{D}, \widehat{P})=\operatorname{ind}\left(\widehat{D}, \widehat{P}_{+}\right)+\operatorname{ind}\left(\widehat{B}: \widehat{P}_{+} \mathcal{H}_{m}^{s-1 / 2}(Y) \rightarrow \mathcal{L}\right) .
$$

Problem (3.10) is Fredholm if and only if the principal symbol $B$ of the operator $\widehat{B}$ is an isomorphism between the principal symbol $L_{+}$of the subspace $\widehat{L}_{+}=\widehat{P}_{+} \mathcal{H}_{m}^{s-1 / 2}(Y)$ and $L$. In this case, the above general index formula (3.16) is valid.

\subsection{Model boundary value problems on the cylinder}

Applications of the locality principle and the corresponding surgery to boundary value problems, given later on in this section, use model boundary value problems on the cylinder as the simplest model to which more general problems are reduced by surgery. In this subsection, we consider these model problems.

Let $Y$ be a closed $C^{\infty}$ manifold. On the cylinder

$$
C=Y \times[-1,1]
$$

with boundary

$$
\partial C=(Y \times\{-1\}) \cup(Y \times\{+1\})
$$

consisting of two separate components (faces) $Y \times\{ \pm 1\}$, we consider an elliptic differential operator $D$ of order $m$ with coefficients independent of the coordinate $t \in[-1,1]$ :

$$
\widehat{D}=\left(-i \frac{\partial}{\partial t}\right)^{m}+\sum_{j=0}^{m-1} \widehat{D}_{j}\left(-i \frac{\partial}{\partial t}\right)^{j}
$$

Here $\widehat{D}_{j}$ is a differential operator of order $m-j$ on $Y$; in accordance with the preceding, we assume that $\widehat{D}_{m}$ (the coefficient of $\left.(-i \partial / \partial t)^{m}\right)$ is the identity operator.

The conormal symbol of $\widehat{D}$ on each of the faces has the form

$$
\begin{gathered}
\mathfrak{D}_{-1}(p)=p^{m}+\sum_{j=0}^{m-1} \widehat{D}_{j} p^{j} \quad \text { on } \quad Y \times\{-1\}, \\
\mathfrak{D}_{1}(p)=\mathfrak{D}_{-1}(-p) \equiv(-p)^{m}+\sum_{j=0}^{m-1} \widehat{D}_{j}(-p)^{j} \quad \text { on } \quad Y \times\{1\} .
\end{gathered}
$$


We denote $\mathfrak{D}_{-1}(p)$ simply by $\mathfrak{D}(p)$ and the corresponding positive spectral projection in $\mathcal{H}_{m}^{s}(Y)$ by $\widehat{P}_{+}$. Then the positive spectral projection corresponding to $\mathfrak{D}_{1}(p)$ differs from $\widehat{P}_{-}=1-\widehat{P}_{+}$by a finite-dimensional operator (and coincides with $\widehat{P}_{-}$if $D(p)$ is invertible for all $p \in \mathbb{R})$.

\section{Model problem 1 (a spectral problem)}

$$
\left\{\begin{aligned}
\widehat{D} u & =f \in H^{s}(C), \\
\widehat{P}_{+} j_{Y \times\{-1\}}^{m-1} u & =g \in \widehat{P}_{+} \mathcal{H}_{m}^{s-1 / 2}(Y), \\
\widehat{P}_{-} j_{Y \times\{1\}}^{m-1} u & =h \in \widehat{P}_{-} \mathcal{H}_{m}^{s-1 / 2}(Y) .
\end{aligned}\right.
$$

In this problem, the boundary conditions are determined by complementary projections $\left(\widehat{P}_{+}+\widehat{P}_{-}=1\right)$ on the faces of the cylinder.

Theorem 3.1. The index of the model problem (3.21) is zero.

\section{Model problem $\mathbf{1}^{\prime}$ (a spectral problem)}

$$
\left\{\begin{aligned}
\widehat{D} u & =f \in H^{s}(C), \\
\widehat{P}_{-1} j_{Y \times\{-1\}}^{m-1} u & =g \in \widehat{P}_{-1} \mathcal{H}_{m}^{s-1 / 2}(Y), \\
\widehat{P}_{1} j_{Y \times\{+1\}}^{m-1} u & =h \in \widehat{P}_{1} \mathcal{H}_{m}^{s-1 / 2}(Y) .
\end{aligned}\right.
$$

Here $\widehat{P}_{-1}$ and $\widehat{P}_{1}$ are arbitrary pseudodifferential projections with the same principal symbols as $\widehat{P}_{+}$and $\widehat{P}_{-}$, respectively (say, the Calderón projections).

Theorem 3.2. The index of the model problem (3.21) is equal to

$$
\operatorname{ind}\left(\widehat{D}, \widehat{P}_{-1}, \widehat{P}_{1}\right)=\operatorname{ind}\left(\widehat{P}_{+}, \widehat{P}_{-1}\right)+\operatorname{ind}\left(\widehat{P}_{-}, \widehat{P}_{1}\right)
$$

where $\operatorname{ind}(\widehat{P}, \widehat{Q})$ is the relative index of two projections $\widehat{P}$ and $\widehat{Q}$ differing by a compact operator.

Proof. This is a special case of the general formula (3.16).

Model problem 2 (a classical problem)

$$
\left\{\begin{aligned}
\widehat{D} u & =f \in H^{s}(C), \\
\widehat{B}_{-1} j_{Y \times\{-1\}}^{m-1} u & =g \in H^{k}\left(Y, F_{-1}\right), \\
\widehat{B}_{1} j_{Y \times\{1\}}^{m-1} u & =h \in H^{l}\left(Y, F_{1}\right),
\end{aligned}\right.
$$

where $\widehat{B}_{-1}$ and $\widehat{B}_{1}$ are operators of classical boundary conditions on the faces of the cylinder satisfying the Shapiro-Lopatinskii conditions and $F_{-1}$ and $F_{1}$ are some bundles over $Y$. 
Theorem 3.3. The index of the model problem 2 is equal to

$$
\operatorname{ind}\left(\widehat{D}, \widehat{B}_{-1}, \widehat{B}_{1}\right)=\text { ind } \widehat{\mathcal{B}} \text {, }
$$

where $\widehat{\mathcal{B}}$ is an elliptic operator on $Y$ in the spaces

$$
\widehat{\mathcal{B}}: \mathcal{H}_{m}^{s-1 / 2}(Y) \rightarrow H^{k}\left(Y, F_{-1}\right) \oplus H^{l}\left(Y, F_{1}\right)
$$

with principal symbol

$$
\begin{aligned}
& B_{1} \quad L_{+} \quad \pi^{*} F_{-1} \\
& \mathcal{B}=\oplus: \oplus \longrightarrow \oplus \\
& B_{2} \quad L_{-} \quad \pi^{*} F_{1}
\end{aligned}
$$

Here $L_{+}$and $L_{-}$are the ranges of the principal symbols of the projections $\widehat{P}_{+}$and $\widehat{P}_{-}$ (the Calderón bundles), i.e., the principal symbols of the spaces $\widehat{L}_{+}$and $\widehat{L}_{-}$.

Proof. Theorem 3.3 follows from formula (3.16) applied to problems (3.21) and (3.24) with regard to Theorem 3.1 .

\subsection{The Agranovich-Dynin theorem}

This theorem, as well as the 'dual' Agranovich theorem considered in the next subsection, expresses the locality principle as applied to boundary value problems. Both theorems were obtained at very early stages of the development of index theory of boundary value problems. The history of the topic is described in [2], where one can also find references to the original papers.

Theorem 3.4. Let $\widehat{D}$ be an elliptic differential operator on a compact $C^{\infty}$ manifold $X$ with boundary $\partial X=Y$, and let $\widehat{B}_{1}$ and $\widehat{B}_{2}$ be two operators each of which specifies elliptic (in the sense of Shapiro-Lopatinskii) boundary conditions for the operator $\widehat{D}$. Then the relative index of the elliptic boundary value problems $\left(\widehat{D}, \widehat{B}_{1}\right)$ and $\left(\widehat{D}, \widehat{B}_{2}\right)$ is equal to

$$
\operatorname{ind}\left(\widehat{D}, \widehat{B}_{1}\right)-\operatorname{ind}\left(\widehat{D}, \widehat{B}_{2}\right)=\operatorname{ind}\left(\widehat{B_{1} \circ B_{2}^{-1}}\right)
$$

where $\left(\widehat{B_{1} \circ B_{2}^{-1}}\right)$ is an elliptic $\Psi D O$ on $Y$ with principal symbol $\left(B_{1} \circ B_{2}^{-1}\right)$; here $B_{1}$ and $B_{2}$ are treated as the restrictions of the principal symbols of the operators $\widehat{B}_{1}$ and $\widehat{B}_{2}$ to the subbundle $L_{+}$, which is the principal symbol of the subspace $\widehat{L}_{+}$.

Proof. We shall derive this well-known theorem from the locality principle for the relative index. We equip Sobolev spaces on $X$ with the structure of bottleneck spaces using a function $\chi: X \rightarrow[-1,1]$ equal to -1 in a neighborhood of $Y$, equal to 1 outside the collar neighborhood $U$ of $Y$, and increasing from -1 to 1 in $U$. In various function spaces on $Y$, we also introduce the structure of bottleneck spaces by setting

$$
\varphi g \stackrel{\text { def }}{=} \varphi(-1) g
$$


for any elements $g$ of such spaces and any $\varphi \in C^{\infty}([-1,1])$. Then elliptic boundary value problems generate elliptic operators in bottleneck spaces (this follows from the structure of parametrices of boundary value problems; e.g., see [17]). Without loss of generality, we can assume that the coefficients of $\widehat{D}$ are independent of the collar variable $t$ in $U$. Consider the diagram of modifications shown in Fig. 3.1. Here $\widehat{D}_{0}$ in the right column

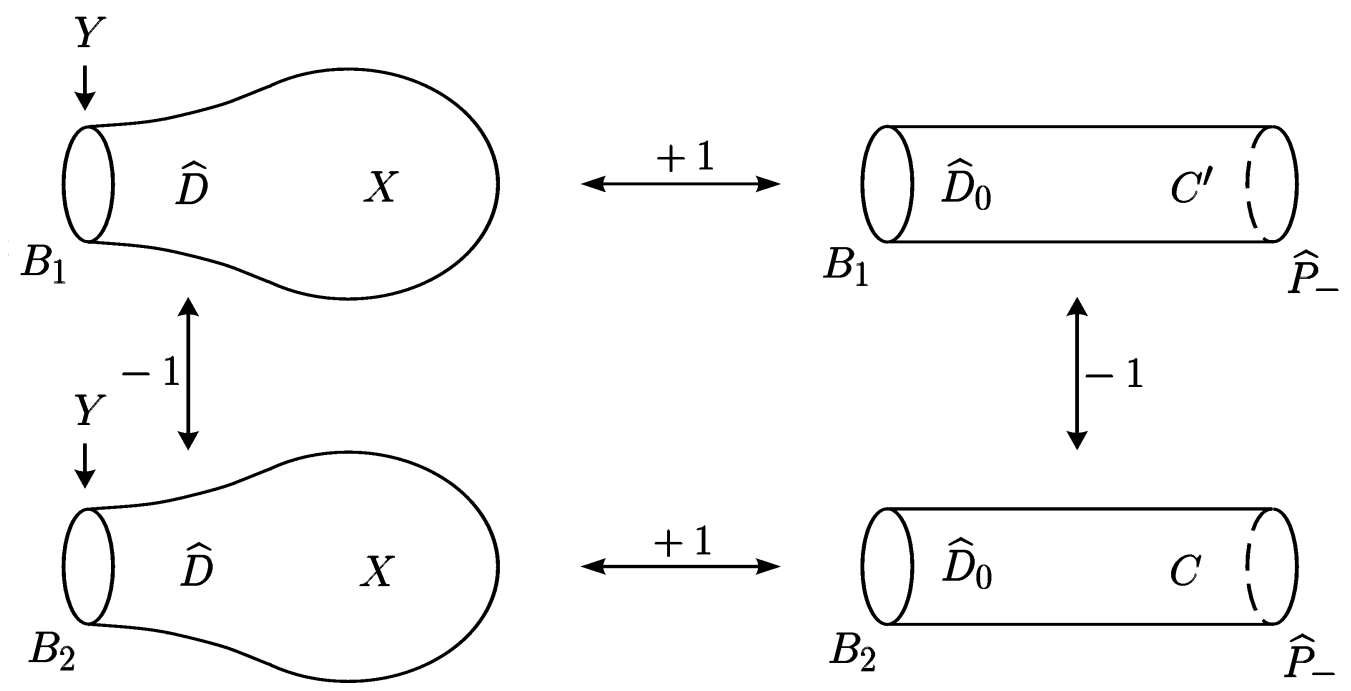

Figure 3.1: Modifications for boundary value problems.

of the diagram is the operator on the cylinder naturally obtained from $\widehat{D}$ by freezing the coefficients on the boundary.

By the locality principle for the relative index, we have

$$
\operatorname{ind}\left(\widehat{D}, \widehat{B}_{1}\right)-\operatorname{ind}\left(\widehat{D}, \widehat{B}_{2}\right)=\operatorname{ind}\left(\widehat{D}_{0}, \widehat{B}_{1}, \widehat{P}_{-}\right)-\operatorname{ind}\left(\widehat{D}_{0}, \widehat{B}_{2}, \widehat{P}_{-}\right)
$$

The indices on the right-hand side can be computed by formula (3.16) with regard to the fact that the index of the problem $\left(\widehat{D}_{0}, \widehat{P}_{+}, \widehat{P}_{-}\right)$is zero. We have

$$
\begin{aligned}
& \operatorname{ind}\left(\widehat{D}_{0}, \widehat{B}_{1}, \widehat{P}_{-}\right)=\operatorname{ind}\left(\widehat{B}_{1}: \widehat{L}_{+} \rightarrow \mathcal{L}_{1}\right), \\
& \operatorname{ind}\left(\widehat{D}_{0}, \widehat{B}_{2}, \widehat{P}_{-}\right)=\operatorname{ind}\left(\widehat{B}_{2}: \widehat{L}_{+} \rightarrow \mathcal{L}_{2}\right),
\end{aligned}
$$

where $\mathcal{L}_{1}$ and $\mathcal{L}_{2}$ are the Sobolev spaces on $Y$ in which the operators $\widehat{B}_{1}$ and $\widehat{B}_{2}$ act. Then

$$
\operatorname{ind}\left(\widehat{D}_{0}, \widehat{B}_{1}, \widehat{P}_{-}\right)-\operatorname{ind}\left(\widehat{D}_{0}, \widehat{B}_{2}, \widehat{P}_{-}\right)=\operatorname{ind}\left(\widehat{B}_{1} \widehat{B}_{2}^{[-1]}: \mathcal{L}_{2} \rightarrow \mathcal{L}_{1}\right)=\operatorname{ind}\left(\widehat{B_{1} B_{2}^{-1}}\right)
$$

as desired. (By $\widehat{B}_{2}^{[-1]}$ we denote the almost inverse of $\widehat{B}_{2}$.) 


\subsection{The Agranovich theorem}

The Agranovich theorem deals in a sense with the opposite case.

Theorem 3.5. Let $\widehat{D}_{1}$ and $\widehat{D}_{2}$ be two elliptic dimensional operators on a compact $C^{\infty}$ manifold $X$ with boundary $\partial X=Y$ coinciding in a collar neighborhood of the boundary, and let $\widehat{B}$ be a boundary operator satisfying the Shapiro-Lopatinskii conditions with respect to $\widehat{D}_{1}$ (and hence with respect to $\left.\widehat{D}_{2}\right)$. Then the relative index of the problems $\left(\widehat{D}_{1}, \widehat{B}\right)$ and $\left(\widehat{D}_{2}, \widehat{B}\right)$ is equal to

$$
\operatorname{ind}\left(\widehat{D}_{1}, \widehat{B}\right)-\operatorname{ind}\left(\widehat{D}_{2}, \widehat{B}\right)=\operatorname{ind}\left(\widehat{D_{1} D_{2}^{-1}}\right),
$$

where $\widehat{D_{1} D_{2}^{-1}}$ is a $\Psi D O$ on $X$ with principal symbol $D_{1} D_{2}^{-1}$ acting as the identity operator of functions supported in a sufficiently small neighborhood of the boundary.

Remark 3.6. The operator $D_{1} D_{2}^{-1}$ obviously requires no boundary conditions.

Proof. The operators $\widehat{D}_{1}$ and $\widehat{D}_{2}$ can be extended to the double $2 X=X \cup_{Y} X$ as elliptic operators (see [41]).

Since $\widehat{D}_{1}$ and $\widehat{D}_{2}$ coincide near the boundary, we can assume that the extensions coincide on the second copy of $X$. Let us denote these extensions by $\mathfrak{D}_{1}$ and $\mathfrak{D}_{2}$. Now consider the modification diagram shown in Fig. 3.2.

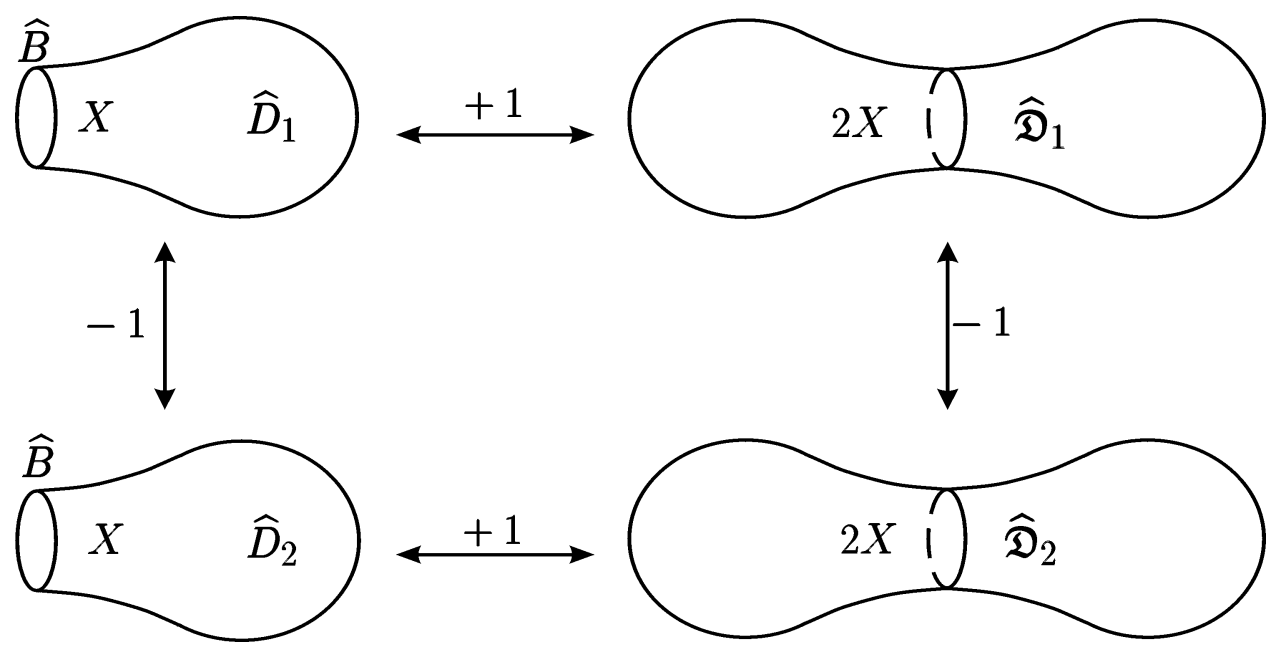

Figure 3.2: Extension to the double.

By the locality principle for the relative index, we obtain

$$
\operatorname{ind}\left(\widehat{D}_{1}, \widehat{B}\right)-\operatorname{ind}\left(\widehat{D}_{2}, \widehat{B}\right)=\operatorname{ind}\left(\widehat{\mathfrak{D}}_{1}\right)-\operatorname{ind}\left(\widehat{\mathfrak{D}}_{2}\right)=\operatorname{ind}\left(\widehat{\mathfrak{D}_{1} \mathfrak{D}_{2}^{-1}}\right) .
$$


But it is obvious that

$$
\operatorname{ind}\left(\widehat{\mathfrak{D}_{1} \mathfrak{D}_{2}^{-1}}\right)=\operatorname{ind}\left(\widehat{D_{1} D_{2}^{-1}}\right)
$$

since the symbol $\mathfrak{D}_{1} \mathfrak{D}_{2}^{-1}$ of the operator $\widehat{\mathfrak{D}_{1} \mathfrak{D}_{2}^{-1}}$ is equal to unity on the second copy of $X$ and in a neighborhood of $Y$, so that this operator can be homotopied to an operator acting as the identity operator on functions supported on the second copy of $X$ or in a neighborhood of $Y$.

\subsection{The Bojarski theorem and its generalizations}

In the mid-seventies, Bojarski put forward the following cutting conjecture in the framework of a surgery proof of the Atiyah-Singer index theorem, which he was developing at the time. Consider a Dirac operator $\widehat{D}$ on a closed connected manifold $M$. We cut $M$ by a two-sided hypersurface $S$ into two parts $M_{+}$and $M_{-}, \partial M_{+}=\partial M_{-}=S$, and equip the resulting Dirac operators on $M_{+}$and $M_{-}$with the Atiyah-Patodi-Singer conditions $\widehat{P}_{+} u_{+}=0, \widehat{P}_{-} u_{-}=0$. Then the index of the Dirac operator on $M$ is equal to the relative index of the Fredholm pair of subspaces

$$
\left(\widehat{L}_{+}=\operatorname{Im} \widehat{P}_{+}, \widehat{L}_{-}=\operatorname{Im} \widehat{P}_{-}\right) .
$$

Later, this conjecture was proved (the Bojarski theorem); see the book [8] for details. Here we shall prove a theorem on cutting an arbitrary elliptic operator into boundary value problems.

Let $M$ be a closed $C^{\infty}$ manifold, $\widehat{D}$ an elliptic differential operator on $M$, and $S \subset M$ a smooth two-sided hypersurface. We cut $M$ along $S$ into two manifolds $M_{+}$and $M_{-}$ with boundary $\partial M_{+}=\partial M_{-}=S$ and consider general elliptic boundary value problems on $M_{+}$and $M_{-}$:

$$
\begin{aligned}
& \left\{\begin{aligned}
\widehat{D} u_{+} & =f_{+}, \quad \text { on } \quad M_{+}, \\
\widehat{B}_{+} j_{S}^{m-1} u_{+} & =g_{+} \in \mathcal{L}_{+},
\end{aligned}\right. \\
& \left\{\begin{aligned}
\widehat{D} u_{+} & =f_{-}, \quad \text { on } \quad M_{-}, \\
\widehat{B}_{-} j_{S}^{m-1} u_{-} & =g_{-} \in \mathcal{L}_{-},
\end{aligned}\right.
\end{aligned}
$$

where

$$
\begin{aligned}
& \widehat{B}_{+}: \mathcal{H}_{m}^{s}(S) \rightarrow \mathcal{L}_{+} \\
& \widehat{B}_{-}: \mathcal{H}_{m}^{s}(S) \rightarrow \mathcal{L}_{-},
\end{aligned}
$$

are some operators of boundary conditions such that problems (3.33) and (3.34) are Fredholm and $\mathcal{L}_{+}$and $\mathcal{L}_{-}$are some Hilbert spaces. The restrictions of $\widehat{B}_{+}$to $\widehat{L}_{+}$and $\widehat{B}_{-}$ to $\widehat{L}_{-}$, where $\widehat{L}_{ \pm}=\operatorname{Im} \widehat{P}_{ \pm}$and the projections $\widehat{P}_{ \pm}$correspond to the conormal symbol of the operator $\widehat{D}$, will be denoted by the same letters. 
Theorem 3.7.

$\operatorname{ind} \widehat{D}=\operatorname{ind}\left(\widehat{D}_{+}, \widehat{B}_{+}\right)+\operatorname{ind}\left(\widehat{D}_{-}, \widehat{B}_{-}\right)-\operatorname{ind}\left(\begin{array}{ccccc}\widehat{B}_{+} & \widehat{L}_{+} & & \mathcal{L}_{+} \\ \oplus & : & \oplus & \longrightarrow & \oplus \\ \widehat{B}_{-} & \widehat{L}_{-} & & \mathcal{L}_{-}\end{array}\right)$

Proof. Consider the modification diagram shown in Fig. 3.3.

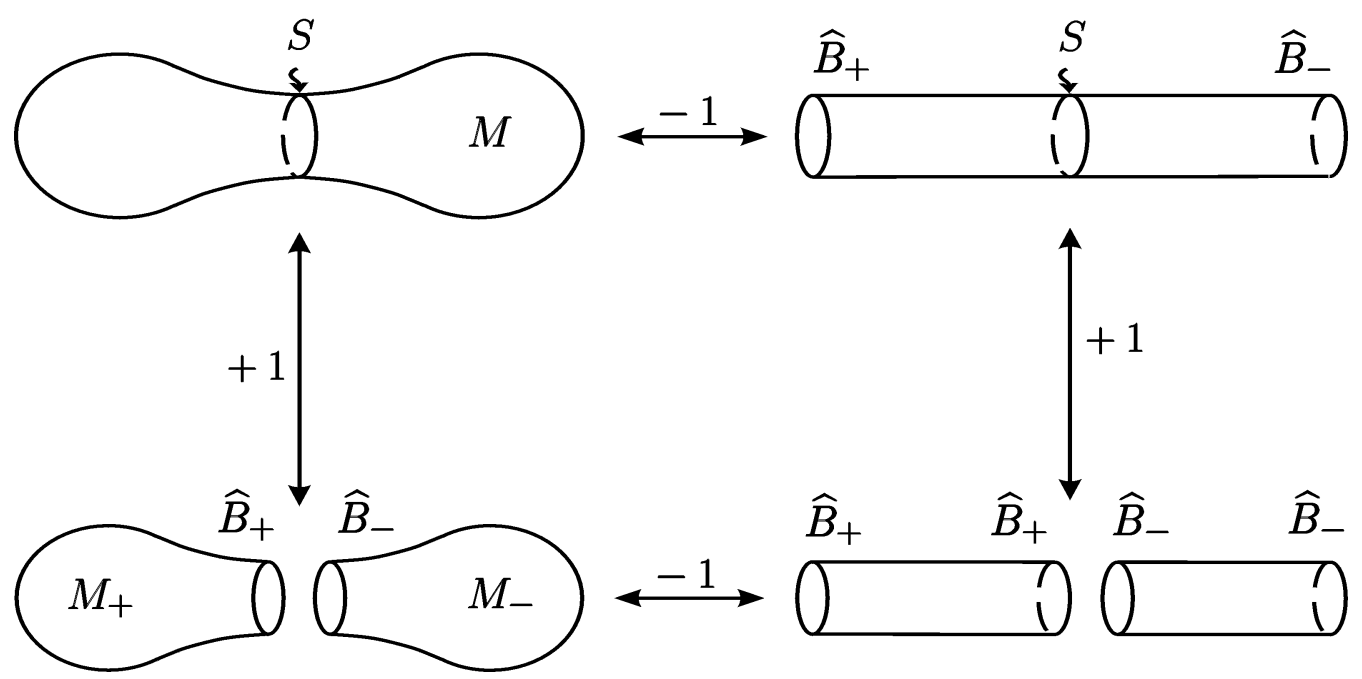

Figure 3.3: Cutting into boundary value problems.

In the left column, the main elliptic operator is the operator $\widehat{D}$ on $M$ and its restrictions to $M_{+}$and $M_{-}$. In the right column, the main operator is given by the extension to the finite cylinder $C$ of the operator $\widehat{D}$ with coefficients freezed on $S$. Needless to say, just as before, we assume that the coefficients of $\widehat{D}$ are independent of the collar variable $t$. By the relative index theorem, we have

$$
\text { ind } \widehat{D}-\operatorname{ind}\left(\widehat{D}_{+}, \widehat{B}_{+}\right)-\operatorname{ind}\left(\widehat{D}_{-}, \widehat{B}_{-}\right)=\operatorname{ind} \widehat{B}_{+} \oplus \widehat{B}_{-}
$$

(the remaining two terms on the right-hand side are zero). The proof of the theorem is complete. 


\subsection{Boundary value problems with symmetric conormal symbol}

On a $C^{\infty}$ manifold $X$ with boundary $\partial X=Y$, we consider an elliptic boundary value problem

$$
\left\{\begin{array}{l}
\widehat{D} u=f, \\
\widehat{B} j_{Y}^{m-1} u=g \in \mathcal{L} .
\end{array}\right.
$$

Suppose that the conormal symbol $\widehat{D}_{0}(p)$ of the operator

$$
\widehat{D}: H^{s}(X, E) \rightarrow H^{s-m}(X, F)
$$

satisfies the symmetry condition

$$
\widehat{D}_{0}(p)=\widehat{D}_{0}(-p)
$$

(One can also consider the more general case in which the symmetry includes a diffeomorphism $g: Y \rightarrow Y$ and associated bundle isomorphisms

$$
\left.\mu_{E}:\left.\left.E\right|_{Y} \rightarrow g^{*} E\right|_{Y}, \quad \mu_{F}:\left.\left.F\right|_{Y} \rightarrow g^{*} F\right|_{Y}\right) .
$$

In this case, using the general index locality principle, for the index of the boundary value problem (3.38) one can give a simpler formula than the general Atiyah-Bott formula $[4]$.

Indeed, consider the surgery that takes two copies of the operator $\left\{\widehat{D}, \widehat{B} j_{Y}^{n-1}\right\}$ to a new operator $\widehat{\widetilde{D}}$ on the closed manifold (double) $2 M$ and the operator $\widehat{D}_{0}=\widehat{D}_{0}(-i \partial / \partial t)$ with coefficients independent of $t$ on the cylinder $Y \times[-1 / 2,1 / 2]$ with the boundary conditions

$$
\begin{aligned}
& \widehat{B} j_{\{-1 / 2\}}^{m-1} u=g_{1}, \\
& \widehat{B} j_{\{+1 / 2\}}^{m-1} u=g_{2}
\end{aligned}
$$

on the faces.

The index of this model boundary value problem on the cylinder is equal to the index of the spectral problem for $\widehat{D}_{0}$ plus the index of the operator

$$
\left(\begin{array}{c}
\widehat{B} \\
\widehat{B}
\end{array}\right): \begin{aligned}
& \widehat{L}_{+} \\
& \widehat{L}_{-}
\end{aligned} \rightarrow \begin{aligned}
& \mathcal{L} \\
& \bigoplus \\
& \mathcal{L}
\end{aligned}
$$

where $\widehat{L}_{ \pm} \subset \bigoplus_{k=0}^{m-1} H^{s-1 / 2-k}\left(Y,\left.E\right|_{Y}\right)$ are the Calderón subspaces [9] corresponding to the left and right faces of the cylinder. Without loss of generality (say, adding a constant 
to $\widehat{D}$, which does not affect the index) we can assume that $\widehat{D}_{0}(p)$ is invertible for real $p$. Then the index of the spectral boundary value problem for $\widehat{D}_{0}$ is zero and

$$
\widehat{L}_{+} \oplus \widehat{L}_{-}=\bigoplus_{k=0}^{m-1} H^{s-1 / 2-k}\left(Y,\left.E\right|_{Y}\right)
$$

by the symmetry condition. Finally, we obtain the following theorem.

Theorem 3.8. The index formula

$$
\operatorname{ind}\left(\widehat{D}, \widehat{B} \circ j_{Y}^{m-1}\right)=\frac{1}{2}\left\{\operatorname{ind}(\widehat{\widetilde{D}})+\operatorname{ind}\left(\widehat{B} \oplus \widehat{B}: \bigoplus_{k=0}^{m-1} H^{s-\frac{1}{2}-k}\left(Y,\left.E\right|_{Y}\right) \longrightarrow \mathcal{L} \oplus \mathcal{L}\right)\right\}
$$

is valid.

If the boundary value problem (3.38) is classical, then both terms in this index formula are the indices of elliptic operators on closed manifolds. The exposition in this subsection generally follows $[37,38]$.

\section{Localization in index theory on singular manifolds}

\subsection{The index of elliptic pseudodifferential operators}

We freely use the notation and results of the theory of differential equations on manifolds with singularities (e.g., see [38] and references therein).

\subsubsection{Index on manifolds with cuspidal singularities}

Here we show that computing the index on manifolds with cuspidal singularities can always be reduced to the conical case.

Let $M$ be a manifold with singularities $\alpha_{1}, \ldots \alpha_{N}$ of cuspidal type of orders $k_{1}, \ldots, k_{N}$, respectively. (We do not exclude the case in which some $k_{j}=0$, i.e., the corresponding points are conical), and let $M^{\prime}$ be the corresponding manifold with conical singularities $\alpha_{1}, \ldots, \alpha_{N}$. This means that the underlying manifold with isolated singularities is the same for $M$ and $M^{\prime}$, and only the additional structures differ.

Theorem 4.1 (e.g., see [38]). Let $\widehat{D}$ be an elliptic $\Psi D O$ in the Sobolev spaces $H_{k}^{s, \gamma}(M)$ on the manifold $M$ with principal symbol $\sigma(\widehat{D})$ and conormal symbol $\widehat{D}_{0}(p)=\sigma_{c}(\widehat{D})$, and let $\widehat{D}^{\prime}$ be an elliptic $\Psi D O$ in the Sobolev spaces $H^{s, \gamma}\left(M^{\prime}\right)$ on $M^{\prime}$ with the same principal and conormal symbols. Then

$$
\text { ind } \widehat{D}=\text { ind } \widehat{D}^{\prime} \text {. }
$$

With this theorem in mind, in all subsequent exposition we consider only elliptic $\Psi$ DO on manifolds with conical singularities. 


\subsubsection{The relative index formula}

Let $M$ be a manifold with conical singularities $\alpha_{1}, \ldots, \alpha_{N}$ (the bases of the corresponding cones will be denoted by $\Omega_{1}, \ldots, \Omega_{N}$ ), and let an elliptic principal symbol $D$ of some order $m$ on $T^{*} M \backslash\{0\}$ and conormal symbols $D_{j}(p)$ on $\Omega_{j}, j=1, \ldots, N$, satisfying the matching conditions

$$
\sigma\left(D_{j}(p)\right)=\left.D\right|_{\partial T^{*} M}, \quad j=1, \ldots, N,
$$

be given. Suppose that $D_{j}(p)$ depends on $p$ analytically and is and elliptic $\Psi$ DO with parameter $p$ in the sense of Agranovich-Vishik in the strip $a_{j}<\operatorname{Im} p<b_{j}$. For every vector $\gamma=\left(\gamma_{1}, \ldots, \gamma_{N}\right)$ of weight exponents such that $\gamma_{j} \in\left(a_{j}, b_{j}\right), j=1, \ldots, N$, we can uniquely (modulo compact operators of lower order) defined an operator

$$
\widehat{D}_{\gamma}: H^{s, \gamma}(M) \rightarrow H^{s-m, \gamma}(M)
$$

such that

$$
\sigma\left(\widehat{D}_{0}\right)=D, \quad \sigma_{c j}\left(\widehat{D}_{\gamma}\right)=\left.D_{j}(p)\right|_{\mathcal{L}_{\gamma_{j}}}
$$

The operator $\widehat{D}_{\gamma}$ is Fredholm for all $\gamma$ such that none of the lines $\mathcal{L}_{\gamma_{j}}$ contains singular operators of the corresponding conormal trajectory $\sigma_{c j}\left(\widehat{D}_{\gamma}\right)$ (such $\gamma$ are said to be admissible).

The relative index theorem describes the increment

$$
\operatorname{ind}\left(\widehat{D}_{\widetilde{\gamma}}\right)-\operatorname{ind}\left(\widehat{D}_{\gamma}\right)
$$

in the index of this elliptic operator in the passage from one admissible $\gamma$ to another.

One can readily see that the increment (4.4) is the sum of increments obtained when only one of the weight exponents varies. Hence we state the theorem for the case in which the weight exponent changes only for one singular point $\alpha$.

Theorem 4.2. Suppose that the conormal symbol $D(p)$ of the operator $\widehat{D}$ at the singular point $\alpha$ is analytic in the strip $a<\operatorname{Im} p<b$. Next, let $\gamma$ and $\widetilde{\gamma} \in(a, b)$ be two admissible values of the weight exponent at $\alpha$, and moreover, $\widetilde{\gamma}>\gamma$. Then

$$
\operatorname{ind}\left(\widehat{D}_{\tilde{\gamma}}\right)-\operatorname{ind}\left(D_{\gamma}\right)=-\sum_{j} \text { Trace } \operatorname{Res}_{p_{j}}\left(D^{-1}(p) \frac{\partial D(p)}{\partial p}\right),
$$

where the sum is taken over all poles $p_{j}$ of the family $D^{-1}(p)$ in the strip $\gamma<\operatorname{Im} p<\widetilde{\gamma}$.

\subsubsection{Statement of the problem}

It was shown in the preceding item that the index of an elliptic operator

$$
\widehat{D}_{\gamma}: H^{s, \gamma}(M) \rightarrow H^{s-m, \gamma}(M),
$$


of order $m$ with given principal and conormal symbols $\sigma(\widehat{D})$ and $\sigma_{c}(\widehat{D})$ on a manifold $M$ with conical singularities in general depends on $\gamma$ (and is independent of $s$ ), and moreover, the relative index, i.e., the increment in the index of $\widehat{D}$ resulting from the variation of $\gamma$ can be expressed via some invariants of the conormal symbol $D(p)=\sigma_{c}(\widehat{D})$, namely, via the multiplicities

$$
m_{j}=\text { Trace } \operatorname{Res}_{p=p_{j}} D(p)^{-1} \frac{\partial D(p)}{\partial p}
$$

of its singular points $p_{j}$, i.e., the poles of the inverse family $D(p)^{-1}$. Hence it is a natural conjecture that the index itself can be represented as the sum of two terms, one of which depends only on the invariants of the conormal symbol (and on $\gamma$ ) and the second only on the stable homotopy class of the principal symbol. Having this in mind, we seek an index formula for the operator (4.6) in the form

$$
\operatorname{ind}\left(\widehat{D}_{\gamma}\right)=f_{1}\left(\left\{m_{j}\right\}, \gamma\right)+f_{2}([\sigma(\widehat{D})]),
$$

where

$$
[\sigma(\widehat{D})] \in K_{c}\left(T^{*} M\right)
$$

is the class of stable homotopy equivalence of the principal symbol in $K$-theory with compact supports on the manifold $T^{*} M$ with boundary. Unfortunately, a formula of the form (4.8) cannot exist in the class of all elliptic $\Psi D O$ on $M$ for deep topological reasons. (For spectral boundary value problems, this was for the first time indicated in [36].)

On the other hand, function (4.8), if it exists, has obvious advantages in that it explicitly separates topological information (which depends only on topological invariants of the principal symbol) and analytical information (depending on analytic invariants of the conormal symbol). In contrast with the case of smooth manifolds, there is no purely topological expression for the index of $\Psi \mathrm{DO}$ on manifolds with singularities. Hence a formula like (4.8), where the inevitable analytical information is isolated and expressed via analytic invariants (multiplicities of singular points) is satisfactory in the situation in question.

Our subsequent program is to obtain conditions under which a formula like (4.8) is possible and find the index formula for the classes of operators satisfying these conditions. The second part of this program has yet been implemented only partly: there is an important class of operators (operators with symmetry conditions) for which the desired formula has been obtained. The problem of finding a formula of the form (4.8) for arbitrary classes of operators satisfying necessary and sufficient conditions for the existence of such a formula remains open.

\subsubsection{The spectral flow}

The notion of spectral flow is well known for families $\left\{A_{t}\right\}$ of self-adjoint elliptic operators (see [7]). It was generalized in [36] to families of normally elliptic operators, which 
permitted considering the problem on invariant index formulas for spectral boundary value problems for differential operators. To analyze elliptic operators on singular manifolds, we need the more general notion of the spectral flow of a family $\left\{D_{t}(p)\right\}$ of conormal symbols elliptic in the sense of Agranovich-Vishik. This notion was introduced in [26, 31], is closely related to some results from [23], and contains the above-mentioned notions as a special case with $D_{t}(p)=p-A_{t}$.

Let $\Omega$ be a smooth compact manifold without boundary, and let $\left\{D_{t}(p)\right\}$ be a family of Agranovich-Vishik elliptic conormal symbols in some strip $|\operatorname{Im} p|<R$, continuously depending on the parameter $t \in[0,1]$. We shall define the notion of spectral flow $\operatorname{sf}\left\{D_{t}(p)\right\}$ of the family $\left\{D_{t}(p)\right\}$ across the real axis (from the open lower half-plane to the closed upper half-plane). We temporarily assume that $D_{0}(p)$ and $D_{1}(p)$ are invertible everywhere on the real axis. For each value of $t$, the family $D_{t}(p)$ has finitely many singular points in the strip $|\operatorname{Im} p|<R / 2$, and the set of singular operators continuously depends on $t$. It follows that there is a finite partition of the interval $[0,1]$ into smaller intervals $\left[t_{i-1}, t_{i}\right]$, $i=1, \ldots, N$, where

$$
0=t_{0}<t_{1}<\ldots<t_{N}=1
$$

and numbers $\gamma_{j}$ such that

(a) $\gamma_{1}=\gamma_{N}=0, \quad\left|\gamma_{i}\right|<R / 2$;

(b) the conormal symbol $D_{t}(p)$ is invertible on the weight $\operatorname{line} \mathcal{L}_{\gamma_{i}}=\left\{\operatorname{Im} p=\gamma_{i}\right\}$ for $t \in\left[t_{i-1}, t_{i}\right]$.

Figure 4.1 shows an example of such a partition.

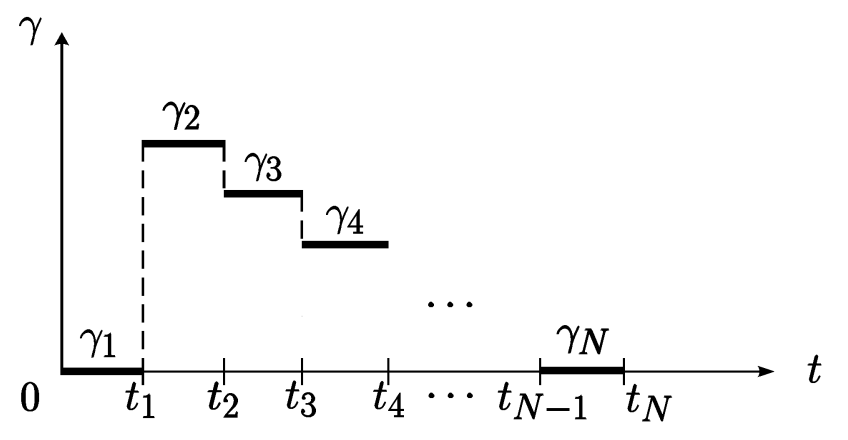

Figure 4.1: Example of a partition.

We define the contribution $l_{i}$ of each point $t_{i}, i=1, \ldots, N-1$, to the spectral flow by the formula

$$
l_{i}= \pm \sum_{j} m\left(p_{j}\right)
$$


where $p_{j}$ are the singular points of $D_{t_{j}}(p)$ between the weight lines $\mathcal{L}_{\gamma_{i}}$ and $\mathcal{L}_{\gamma_{i+1}}$ (note that by condition (b) there are no singular points on the weight lines themselves) and $m\left(p_{j}\right)$ are their multiplicities; the sign "+" or "-" is chosen if $\gamma_{i}<\gamma_{i+1}$ or $\gamma_{i}>\gamma_{i+1}$, respectively.

Theorem 4.3. The sum $\sum_{i=1}^{N-1} l_{i}$ is independent of the choice of the partition $\left\{t_{i}\right\}$ and the numbers $\left\{\gamma_{j}\right\}$ satisfying conditions (a) and (b).

Hence the following is well defined.

Definition 4.4. The spectral flow of the family $\left\{D_{t}(p)\right\}$ is the number

$$
\operatorname{sf}\left\{D_{t}\right\}=\sum_{i} l_{i}
$$

The multiplicities of singular points are always integer, and so is the spectral flow. Now let us remove the restriction that $D_{0}(p)$ and $D_{1}(p)$ must be invertible on the real axis. Namely, we set

$$
\operatorname{sf}\left\{D_{t}\right\}=\lim _{\varepsilon \rightarrow+0} \operatorname{sf}\left\{D_{t}(p-i \varepsilon)\right\} .
$$

For all sufficiently small $\varepsilon>0$, the family $D_{t}(p-i \varepsilon)$ is invertible on the real axis. The spectral flow is continuous and hence is independent of $\varepsilon$, and so to find $\operatorname{sf}\left\{D_{t}\right\}$ one can take a finite value of $\varepsilon$.

The role of the spectral flow in index theory of operators on singular manifolds is determined by the following theorem.

Theorem 4.5 (a generalized relative index formula). Let $\left\{\widehat{D}_{t}\right\}_{t \in[0,1]}$ be a continuous family of formally elliptic operators (i.e., operators with invertible principal symbols) on a manifold $M$ with conical singularities. Suppose that $\widehat{D}_{0}$ and $\widehat{D}_{1}$ are elliptic in $\left\{H^{s, 0}(M)\right\}$ (i.e., the conormal symbols $\sigma_{c}\left(\widehat{D}_{0}\right)$ and $\sigma_{c}\left(\widehat{D}_{1}\right)$ are invertible on the weight line $\mathcal{L}_{0}=$ $\{\operatorname{Im} p=0\})$. Then

$$
\operatorname{ind}\left(D_{0}\right)-\operatorname{ind}\left(D_{1}\right)=\operatorname{sf}\left(\sigma_{c}\left(\widehat{D}_{t}\right)\right) .
$$

From the generalized relative index formula, one can readily derive a criterion for the existence of index formulas of the from (4.8) with splitting into invariant summands.

Theorem 4.6. Let $\mathcal{K}$ be a subclass of the class of formally $\Psi D O$ on a manifold $M$ with conical singularities determined by the condition $\sigma_{c}(\widehat{D}) \in \Sigma$, where $\Sigma$ is a class of conormal symbols. The following assertions are equivalent: (a) for elliptic operators of the class $\mathcal{K}$, there exists an index formula of the form

$$
\text { ind } \widehat{D}=f_{1}+f_{2} \text {, }
$$

where $f_{1}$ depends only on the conormal symbol $\sigma_{c}(\widehat{D})$ and $f_{2}$ depends only on the principal symbol $\sigma(D)$ and is homotopy invariant (in the class $\mathcal{K}$ ); $(b)$ one has

$$
\operatorname{sf}\left\{B_{t}(p)\right\}=0
$$

for an arbitrary periodic family $B_{t} \in \Sigma$. 


\subsubsection{Symmetry conditions and the index formula}

In the preceding item, we established conditions for the existence of a splitting index formula in a given class of elliptic operators on a manifold $M$ with conical singularities. This condition says that the spectral flow must be zero for an arbitrary periodic family of conormal symbols of operators of this class. Since on an arbitrary compact manifold $\Omega$ there is a family of conormal symbols with nonzero spectral flow (an example can be found in [36]), it follows that a splitting formula of the from (4.8) is impossible in the class of all elliptic operators on singular manifolds.

Here we describe an important class of operators for which the formula exists and write out the index formula for operators of that class.

Let $\Omega$ be a smooth compact manifold, and let $E$ and $F$ be vector bundles over $\Omega$. We say that a conormal symbol

$$
D(p): C^{\infty}(\Omega, E) \rightarrow C^{\infty}(\Omega, F)
$$

defined in some strip $|\operatorname{Im} p|<R$ satisfies the symmetry condition [40] if there exists bundle automorphisms

$$
\sigma_{1}: E \rightarrow E, \quad \sigma_{2}: F \rightarrow F
$$

such that

$$
D(-p)=\sigma_{2} D(p) \sigma_{1} \quad \text { for all } p .
$$

Along with condition (4.12), one can consider the slightly more general condition [40]

$$
D\left(p_{0}-p\right)=\sigma_{1} D\left(p_{0}+p\right) \sigma_{2},
$$

obtained from (4.12) by a shift with respect to $p$ (for the corresponding operators on manifolds with conical singularities, condition (4.13) is taken to (4.12) by the transformation

$$
\widehat{D} \mapsto f^{-1} \widehat{D} f,
$$

where $f$ is a nonzero smooth function equal to $r^{p_{0}}$ in a neighborhood of the conical point). This shift and the transformation (4.14) readily reduce all results for (4.13) to the corresponding assertions for (4.12), and so in what follows we consider only condition (4.12).

Let us introduce the objects occurring in the index formula under condition (4.12).

Let $M$ be a manifold with conical singularities. To simplify the statements, we assume that $M$ has a single conical singular point with base $\Omega$. Next, let $\widehat{D}$ be a formally elliptic $\Psi \mathrm{DO}$ on $M$ whose conormal symbol $\sigma_{c}(\widehat{D})(p)$ is defined in the strip $|\operatorname{Im} p|<R$ and satisfies the symmetry condition (4.12) in that strip:

$$
\sigma_{c}(\widehat{D})(-p)=\sigma_{2} \sigma_{c}(\widehat{D})(p) \sigma_{1}
$$

where

$$
\sigma_{1}:\left.\left.E\right|_{\Omega} \rightarrow E\right|_{\Omega}, \quad \sigma_{2}:\left.\left.F\right|_{\Omega} \rightarrow F\right|_{\Omega}
$$


are bundle automorphisms on $\Omega$. (The operator $\widehat{D}$ itself acts in sections of bundles $E$ and $F$ over $M$ ).

Consider the double $2 M$ of $M$. (Figure 4.2 shows the manifold $M$, the blow-up $M^{\wedge}$, and the double $2 M$.) It is constructed as follows. Let $M^{\wedge}$ be the blow-up of $M$; this is a manifold with boundary $\partial M^{\wedge}=\Omega$. We glue two copies of $M^{\wedge}$ together (along the identity map) along $\Omega$ and denote the resulting manifold by $2 M^{\wedge}$. This is a smooth compact manifold without boundary.
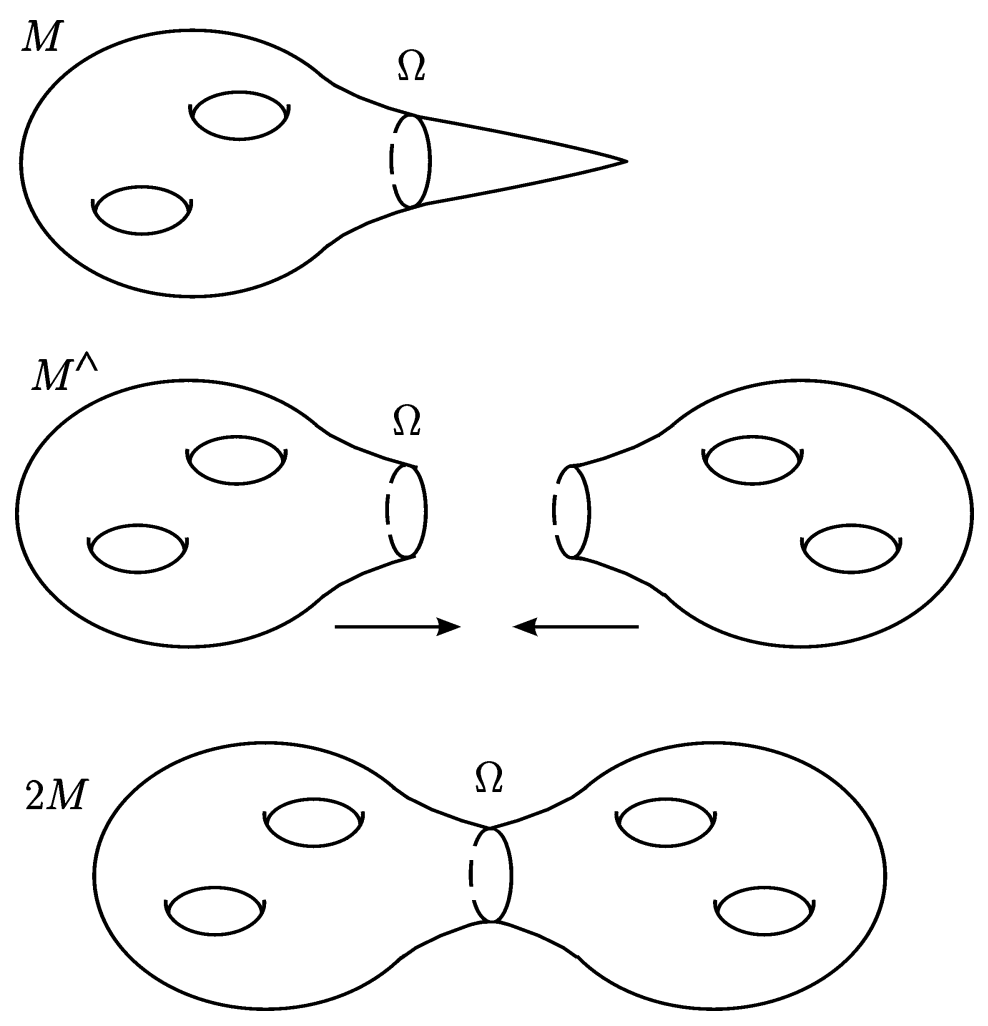

Figure 4.2:

The automorphisms $\sigma_{1}$ and $\sigma_{2}$ permit one to construct the doubles $2 E$ and $2 F$ of the bundles $E$ and $F$. For example, $2 E$ is obtained as the link of two copies of $E$ over respective copies of $M^{\wedge}$ along the mapping $\sigma_{1}$ on $\Omega$ :

$$
2 E=E \bigcup_{\sigma_{1}} E
$$

likewise,

$$
2 F=F \bigcup_{\sigma_{2}} F .
$$


It follows from the symmetry condition (4.15) that the principal symbol

$$
\sigma(\widehat{D}): \pi^{*} E \rightarrow \pi^{*} F
$$

where

$$
\pi: T^{*} M \backslash\{0\} \rightarrow M
$$

is the natural projection, satisfies the symmetry condition

$$
\left.j^{*} \sigma(\widehat{D})\right|_{\partial T^{*} M}=\left.\pi^{*}\left(\sigma_{2}\right) \sigma(\widehat{D})\right|_{\partial T^{*} M} \pi^{*}\left(\sigma_{1}\right),
$$

where

$$
\begin{aligned}
j: \partial T^{*} M & \rightarrow \partial T^{*} M \\
(\omega, p, q) & \mapsto(\omega,-p, q), \quad(\omega, p) \in T^{*} \Omega, \quad p \in \mathbb{R},
\end{aligned}
$$

is the inversion in $\partial T^{*} M$ and $\pi^{*}\left(\sigma_{1}\right)$ and $\pi^{*}\left(\sigma_{2}\right)$ are the natural automorphisms of the bundles $\pi^{*} E$ and $\pi^{*} F$ induced by the automorphisms (4.16). It follows from condition (4.17) that the two copies of the principal symbol are continuously glued into some elliptic symbol

$$
2 \sigma(\widehat{D}): \pi^{*}(2 E) \rightarrow \pi^{*}(2 F),
$$

(where this time $\pi^{*}: T^{*} 2 M \rightarrow 2 M$ ). If in the given trivialization of the collar neighborhood the symbol $\sigma(\widehat{D})$ is independent of $t$ for small $t$, then the symbol (4.18) will be smooth. One can always ensure this by homotopying the operator $\widehat{D}$ with the preservation of the conormal symbol. Throughout the following, we assume that this condition is satisfied. Note that the class of

$$
[2 \sigma(\widehat{D})] \in K_{c}\left(T^{*} 2 M\right),
$$

is independent of the specific choice of the homotopy.

Now we can state the index theorem. It follows from the formal ellipticity condition that the conormal symbol $\sigma_{c}(\widehat{D})$ has a discrete set of singular points in the strip $|\operatorname{Im} p|<R$ and there are finitely many singular points in each proper substrip. For arbitrary $\gamma$, $|\gamma|<R$, such that the weight line $\mathcal{L}_{\gamma}$ does not contain singular points of $\sigma_{c}(\widehat{D})$, the operator

$$
\widehat{D}_{\gamma}: H^{s, \gamma}(M, E) \rightarrow H^{s-m, \gamma}(M, F)
$$

is elliptic.

Theorem 4.7. Under these conditions, one has the index formula

$$
\text { ind } \widehat{D}_{\gamma}=\frac{1}{2}\left\{\text { ind } 2 \widehat{D}-\sum_{\left|\operatorname{Im} p_{j}\right|<\gamma} m_{j}\right\} \text {, }
$$

where $2 \widehat{D}$ is an operator on the closed manifold $2 M$ with principal symbol $2 \sigma(\widehat{D})$ and the $m_{j}$ are the multiplicities of the singular points $p_{j}$ of the conormal symbol $\sigma_{c}(D)$. 
Let us outline the proof of 4.7. (A complete proof by a different method can be found in [40].)

We treat $M$ as a manifold with a cylindrical end, assuming that the coefficients of the operator $\widehat{D}_{\gamma}$ are independent of $t$ for sufficiently large $t$ on the cylindrical end. We also set

$$
\widehat{D}_{0 \gamma}=\sigma_{c}(\widehat{D})\left(-i \frac{\partial}{\partial t}\right): H^{s, \gamma}(C, E) \rightarrow H^{s, \gamma}(C, F),
$$

where $C=\Omega \times(-\infty, \infty)$ is the infinite cylinder. Now consider the following two surgeries shown in Figs. 4.3 and 4.4. (Next to each manifold, the corresponding operator is shown; bottlenecks are dashed.) The surgery shown in Fig. 4.3 is clear; the surgery in 4.4 is just cutting off the left ends of two cylinders and then interchanging them.

Then we have the modification diagram

$$
\begin{aligned}
& \widehat{D}_{\gamma} \oplus \widehat{D}_{-\gamma} \stackrel{1}{\longleftrightarrow} \widehat{D}_{0 \gamma} \oplus \widehat{D}_{0 \gamma} \\
& -1 \uparrow \quad \uparrow-1
\end{aligned}
$$

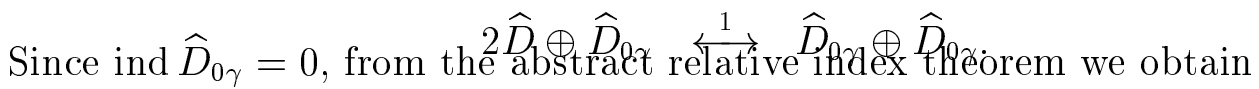

$$
\operatorname{ind}\left(\widehat{D}_{\gamma}\right)+\operatorname{ind}\left(\widehat{D}_{-\gamma}\right)=\operatorname{ind}(2 \widehat{D}) \operatorname{~}
$$

On the other hand, by the relative index theorem,

$$
\operatorname{ind}\left(\widehat{D}_{\gamma}\right)-\operatorname{ind}\left(\widehat{D}_{-\gamma}\right)=-\sum_{\left|\operatorname{Im} p_{j}\right|<\gamma} m_{j}
$$

Solving the simultaneous equations (4.19) and (4.20), we arrive at the assertion of the theorem.

\subsubsection{Historical remarks}

Let us briefly describe the history of symmetry conditions of the form (4.12), which permit one to extend the operator $\widehat{D}$ to the double $2 M$ of the original manifold. Analogs of these conditions for manifolds with boundary occur as early as in the papers $[19,20$, 42] (moreover, they include an involution $g: \Omega \rightarrow \Omega$ reversing the orientation of the boundary), where they were applied to the computation of the signature of a manifold whose boundary admits an involution of this sort. Later, Gilkey and Smith [13, 14] used condition (4.12) for specific operators to compute the eta invariant in some cases. For operators on manifolds with conical singularities, condition (4.12) was introduced in [40]. Later, in [12], the result of [40] was essentially combined with the ideas of $[19,20,42]$ and a symmetry condition including an involution of the base of the cone was considered. The case of general diffeomorphisms $g: \Omega \rightarrow \Omega$ was considered in [25, 26, 31]; the most important feature distinguishing the condition introduced there from all preceding 


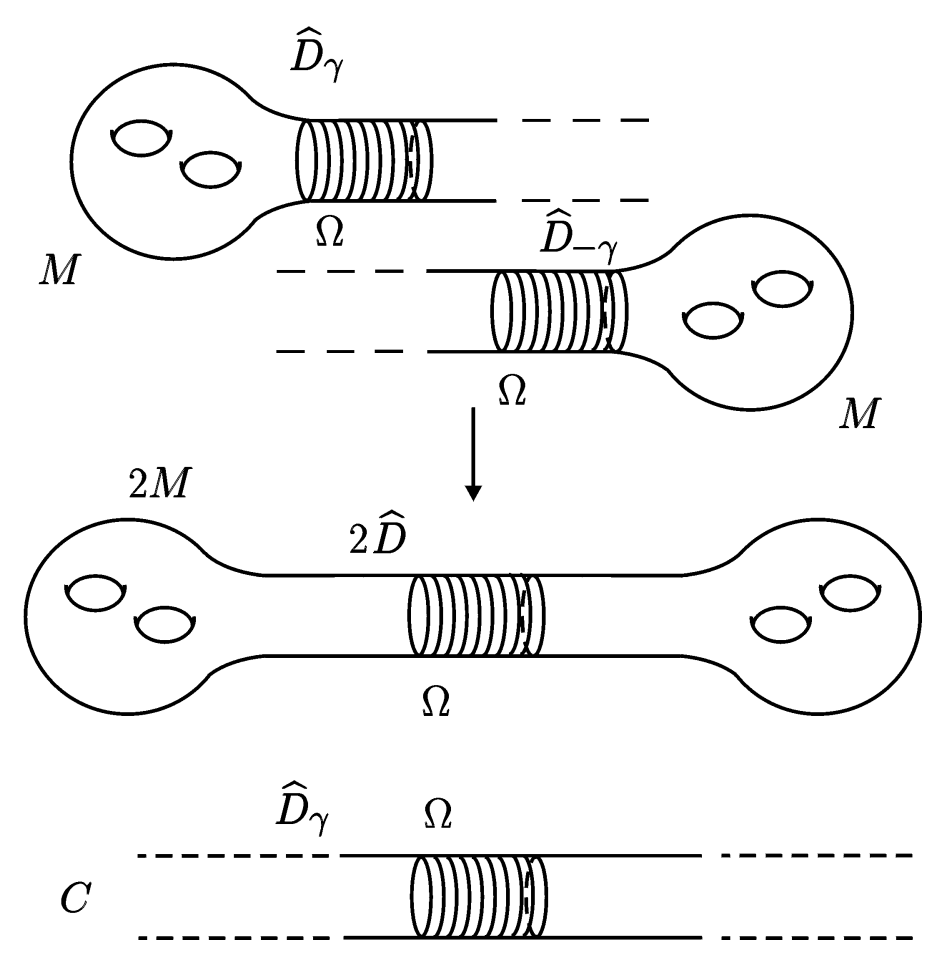

Figure 4.3:

conditions is that it is imposed on the principal symbol of the conormal symbol (that is, the restriction of the interior principal symbol to the boundary $\partial T^{*} M$ ) rather then on the conormal symbol itself.

As to index formulas for operators on singular manifolds without symmetry conditions, we recall the following results.

In [33], an analytic index formula was obtained for $\Psi D O$ on manifolds with conical singularities. Naturally, it comprises three terms, of which the first is expressed via the interior principal symbol, the second is a regularization of the expression

$$
\frac{1}{2 \pi i} \int_{-\infty+i \nu}^{\infty+i \nu} \operatorname{Tr}\left(A^{\prime}(\lambda) A(\lambda)^{-1} d \lambda\right)
$$

where $A(\lambda)$ is the conormal symbol,and the third is responsible for the relative index arising from changes of the weight exponent and is determined by the singularities of the conormal symbol. Next, in $[34,35]$ for the case in which the base of the cone is a 


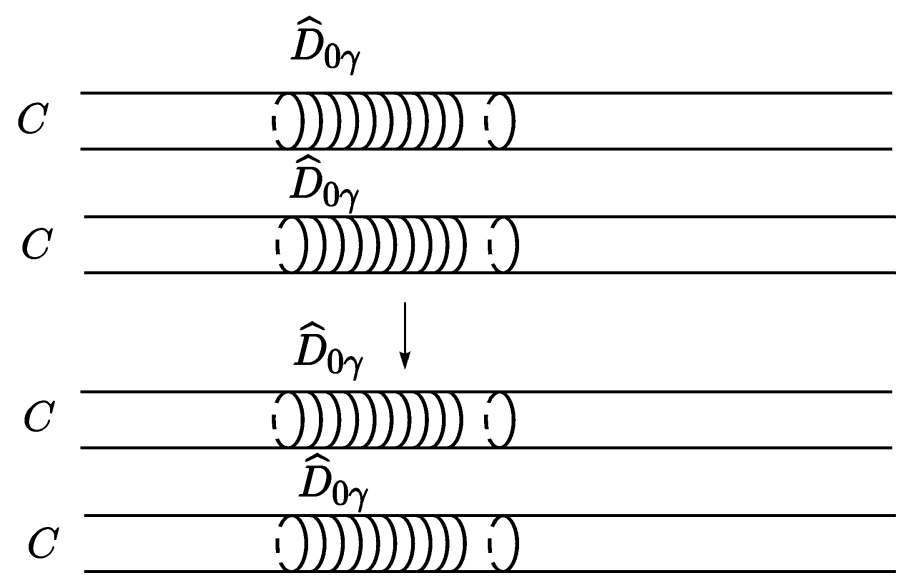

Figure 4.4:

sphere (which can be understood as an operator on a smooth manifold with an isolated discontinuity of the symbol), it was analyzed how one can simplify the index problem by a stable homotopy of symbols, that is, within a single class of the $K_{1}$ group of the symbol algebra. In particular,it was shown that for an odd-dimensional manifold the pair (interior symbol, conormal symbol) can be split, which reduces the problem to two already solved problems, one for the index of an operator on a manifold without singularities and the other for the index of a one-dimensional singular integral operator with operator-valued symbol. In even dimensions, there is a topological obstruction to this homotopy, but it can be removed by an index-preserving surgery. In [35], the case of a symbol that has a discontinuity on a manifold of codimension 1 was considered. Here also a complete splitting was obtained (by a homotopy and an index-preserving surgery) into a nonsingular operator and an operator-valued $\Psi D O$ on the discontinuity manifold.

\subsubsection{Example}

Here we consider an example of an operator satisfying the symmetry conditions. It was partially considered in [40].

The Euler operator on a manifold with conical singularities. Recall (see [32]) that the Euler operator on a smooth manifold $M$ is defined as the operator $d+\delta$ acting from the space of differential forms of even degree on $M$ into the space of differential forms of odd degree. Here $d$ is the exterior differential and $\delta=(-1)^{n p+n+1} * d *$ is the adjoint operator $(*$ is the Hodge operator corresponding to some Riemannian metric.) We use the same definition to construct the Euler operator on a manifold $M$ with conical singularities. For simplicity, we assume that there is only one conical point. In the conical 
neighborhood $U$ of the singular point,

$$
U \cong\{\Omega \times(0,1)\} /\{\Omega \times\{0\}\}
$$

we use the natural conical direct product metric

$$
g=d r^{2}+r^{2} \widetilde{g}
$$

where $\widetilde{g}$ is some Riemannian metric on $\Omega$. For the computations, it is convenient to use the cylindrical coordinates, where the metric acquires the standard form

$$
g=e^{-2 t}\left(d t^{2}+\widetilde{g}\right)
$$

Let $\Lambda^{e v}(U)$ and $\Lambda^{\text {odd }}(U)$ be the bundles of even and odd forms, respectively, on $U$, and let $\Lambda^{e v}(\Omega)$ and $\Lambda^{\text {odd }}(\Omega)$ be the corresponding bundles for the manifold $\Omega$. The direct product structure on $U$ specifies the natural isomorphisms

$$
\begin{aligned}
& \Lambda^{e v}(U) \cong \pi^{*} \Lambda^{e v}(\Omega) \oplus \pi^{*} \Lambda^{\text {odd }}(\Omega), \\
& \Lambda^{\text {odd }}(U) \cong \pi^{*} \Lambda^{\text {odd }}(\Omega) \oplus \pi^{*} \Lambda^{e v}(\Omega),
\end{aligned}
$$

where $\pi: \Omega \times(0,1) \rightarrow \Omega$ is the natural projection on the first factor. These decompositions are obtained as follows: Each form on $U$ is uniquely represented as

$$
\omega=\omega_{1}+d t \wedge \omega_{2}
$$

where ord $\omega_{1}=\operatorname{ord} \omega$, ord $\omega_{2}=\operatorname{ord} \omega-1$, and the forms $\omega_{1}$ and $\omega_{2}$ contain only differentials of coordinates on $\Omega$. The mapping $\omega \mapsto\left(\omega_{1}, \omega_{2}\right)$ specifies the isomorphisms (4.21). Accordingly, $d+\delta$ in $U$ can be represented by the matrix operator

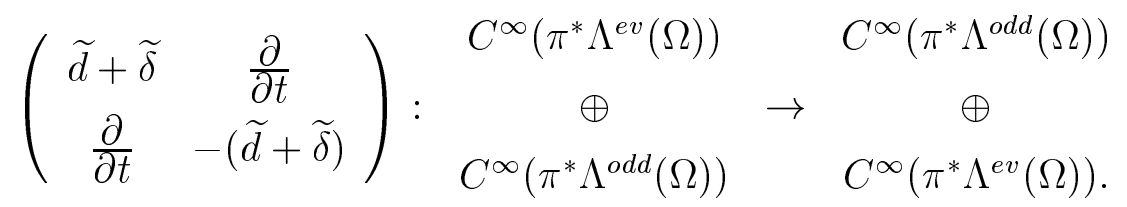

Here $\widetilde{d}$ and $\widetilde{\delta}=(-1)^{(n-1) p+n} \widetilde{*} \widetilde{d} \widetilde{*}$ are the exterior differential and its adjoint on $\Omega$.

The conormal trajectory of the operator (4.24) has the form

$$
D(p)=\left(\begin{array}{cc}
\widetilde{d}+\widetilde{\delta} & i p \\
i p & -(\widetilde{d}+\widetilde{\delta})
\end{array}\right)
$$

and satisfies the symmetry condition

$$
D(-p)=\left(\begin{array}{cc}
1 & 0 \\
0 & -1
\end{array}\right) D(p)\left(\begin{array}{cc}
1 & 0 \\
0 & -1
\end{array}\right)
$$


i.e., the symmetry condition (4.12) with the automorphisms

$$
\sigma_{1}=\sigma_{2}=\left(\begin{array}{cc}
1 & 0 \\
0 & -1
\end{array}\right)
$$

This permits one to compute the index of the Euler operator in an arbitrary scale $H^{s, \gamma}(M)$ provided that $D(p)$ is invertible on the weight line $\mathcal{L}_{\gamma}=\{\operatorname{Im} p=\gamma\}$. To this end, we note that the operator $2 \widehat{D}$ in the index theorem is just the Euler operator on $2 M$.

Hence the answer is

$$
\operatorname{ind}\left(d+\delta: H^{s, \gamma}\left(M, \Lambda^{e v}(M)\right) \rightarrow H^{s, \gamma-1}\left(M, \Lambda^{o d d}(M)\right)=\frac{1}{2}\left(\chi(2 M)-\sum m_{j}\right),\right.
$$

where the $m_{j}$ are the multiplicities of the solution points of the family $D(p)$ in the strip $-\gamma<\operatorname{Im} p<\gamma$. (This formula is valid for $\gamma>0$; for $\gamma<0$, one must take the strip $\gamma<\operatorname{Im} p<-\gamma$, and the terms $m_{j}$ occur in the sum with the opposite sign).

In particular, for small $\gamma$ the conormal symbol $D(p)$ has the unique solution point $p=0$ in this strip. Indeed, there are no other singular points on the real line: if $D(p) u=0$, $u={ }^{t}\left(u_{1}, u_{2}\right)$, then

$$
\begin{aligned}
(\widetilde{d}+\widetilde{\delta}) u_{1}+i p u_{2} & =0, \\
-(\widetilde{d}+\widetilde{\delta}) u_{2}+i p u_{1} & =0
\end{aligned}
$$

and so $($ for $p \neq 0)$

$$
u_{1}=-\frac{i}{p}(\widetilde{d}+\widetilde{\delta}) u_{2}
$$

Substituting $u_{1}$ into the first equation, we obtain

$$
-\frac{i}{p}(\widetilde{d}+\widetilde{\delta})^{2} u_{2}+i p u_{2}=0
$$

or

$$
(\widetilde{d}+\widetilde{\delta})^{2} u_{2}-p^{2} u_{2}=0
$$

i.e.,

$$
\Delta u_{2}=p^{2} u_{2}
$$

where $\Delta$ is the nonpositive Laplacian on $\Omega$. For $p^{2}>0$, this equation has only the trivial solution $u_{2}=0$, and so $u_{1}=0$.

Next,

$$
D(0)=\widetilde{d}+\widetilde{\delta}: \bigoplus C^{\infty}\left(\Lambda ^ { k } ( M ) \rightarrow \bigoplus C ^ { \infty } \left(\Lambda^{k}(M)\right.\right.
$$

is the (total) operator $\widetilde{d}+\widetilde{\delta}$ on $\Omega$. Hence the multiplicity $m_{0}$ of the singular point $p=0$ is equal to

$$
m_{0}=\operatorname{dim} \operatorname{Ker} D(0)=\sum_{j=0}^{n-1} b_{i}(\Omega),
$$


where the $b_{i}(\Omega)$ are the Betti numbers of the manifold $\Omega$. (Recall that the kernel of the operator $\widetilde{d}+\widetilde{\delta}$ coincides with that of the operator $\Delta=(\widetilde{d}+\widetilde{\delta})^{2}$ and consists of harmonic forms on $\Omega$; it remains to apply Hodge theory.) Thus, for sufficiently small $|\gamma|$ we have

$$
\begin{aligned}
\operatorname{ind}(\widetilde{d}+\widetilde{\delta}): H^{s, \gamma} & \left(M, \Lambda^{e v}(M)\right) \rightarrow H^{s, \gamma}\left(M, \Lambda^{\text {odd }}(M)\right) \\
= & \frac{1}{2}\left(\chi(2 M)-\operatorname{sgn} \gamma \cdot \sum_{j=0}^{n-1} b_{j}(\Omega)\right),
\end{aligned}
$$

and the index of the operator $d+\delta$ is expressed in purely topological terms.

\subsection{The index of elliptic Fourier integral operators}

\subsubsection{Statement of the problem}

In the theory of Fourier integral operators (FIO) on smooth manifolds (e.g., see [22], [10], [17], [18], [24], etc.), a distinguished role is played by FIO associated with contact (or homogeneous canonical) transformations, that is, FIO for which the associated Lagrangian manifold in $T^{*}\left(M_{1} \times M_{2}\right) \backslash\{0\}$ is the graph of some (homogeneous) canonical transformation

$$
g: T^{*} M_{1} \backslash\{0\} \rightarrow T^{*} M_{2} \backslash\{0\} .
$$

Namely, such FIO are continuous in the entire Sobolev scale, and their order coincides with the order of the symbol as a homogeneous function. They are elliptic if the symbol does not vanish outside the zero section, and for such operators, Weinstein [44, 45] posed the index problem, which was solved in a special case by Epstein and Melrose [11] and in the general case (for smooth manifolds) by Leichtnam, Nest, and Tsygan [21]. We are interested in the Weinstein problem in the framework of manifold with singularities, and so we deal only with FIO associated with homogeneous canonical transformations of phase spaces where the corresponding configuration spaces have conical singularities. For the structure of classical canonical transformations and the construction of quantized canonical transformations (i.e. Fourier integral operators), we refer the readers to [28].

\subsubsection{The relative index formula}

Just as with $\Psi D O$, we begin the analysis of the index problem by establishing the relative index formula for FIO. Later, in conjunction with symmetry conditions and surgery, this will give the desired general index formula.

Let $T=T(g, a)$ be a formally elliptic FIO on $M$ with conormal symbol $\sigma_{c}(T)=T_{0}(p)$, and let $\gamma_{1}<\gamma_{2}$ be weight exponents such that the weight lines $\mathcal{L}_{\gamma_{1}}$ and $\mathcal{L}_{\gamma_{2}}$ do not contain poles of the family $T_{0}(p)^{-1}$. Then the operators

$$
T_{\gamma_{i}}: H^{s, \gamma_{i}}(H) \rightarrow H^{s-m, \gamma_{i}}(H)
$$


induced by $T$ in the corresponding scales of weighted Sobolev are elliptic and hence Fredholm. Under these conditions, the following theorem holds.

\section{Theorem 4.8 (the relative index formula).}

$$
\operatorname{ind} T_{\gamma_{2}}-\operatorname{ind} T_{\gamma_{1}}=-\sum_{\gamma_{1}<\operatorname{Im} p_{j}<\gamma_{2}} m_{j},
$$

where the $m_{j}$ are the multiplicities of the poles of the family $T_{0}(p)^{-1}$.

Remark 4.9. Formula (4.27) exactly coincides in form with the corresponding formula for $\Psi D O$. This is by no means accidental. We return to the original relative index formula for $\Psi D O$ if $g$ is the identity transformation.

\subsubsection{Symmetry conditions and the index formula}

Here we apply the same technique as in item 4.1.5 to continue an elliptic FIO $T(g, a)$ to the double $2 M$ and, using the index locality principle, obtain a second equation supplementing the relative index formula, so that the resulting system will give the value of ind $T(g, a)$ in terms of the operator on the double $2 M$ and the contribution of the poles of the conormal symbol. Needless to say, this is possible only certain symmetry conditions.

Fourier integral operators in bottleneck spaces. First of all, we shall show that Fourier integral operators on a manifold $M$ with conical singularities can always be viewed as elliptic operators in bottleneck spaces of some special form.

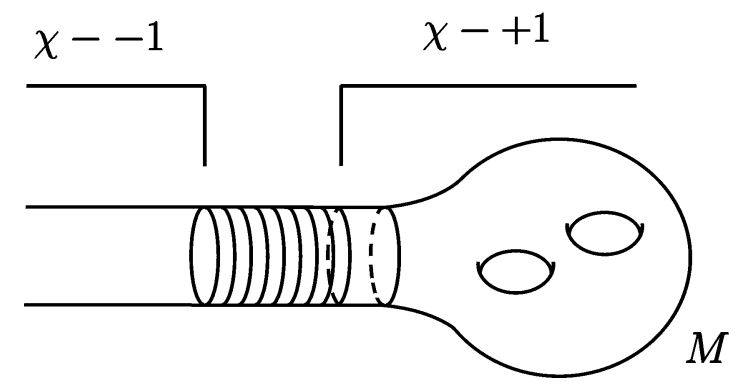

Figure 4.5:

Namely, we treat $M$ as a manifold with a cylindrical end and equip $H^{s, \gamma}(M)$ with the structure of bottleneck spaces by the standard construction: the action of a function $\varphi \in C^{\infty}([-1,1])$ on an element $u(x) \in H^{s, \gamma}(M)$ is reduced to the multiplication by the function $\varphi(\chi(x))$ :

$$
[\varphi-u](x)=\varphi(\chi(x)) u(x)
$$


where $\chi(x)$ is a smooth function growing from -1 to 1 in the bottleneck and constant outside the bottleneck (see Fig. 4.5, where the bottleneck is dashed).

Let $T=T(g, a)$ be an elliptic FIO on $M$. Then it can be included in a continuous family $T(\delta)$ of operators such that

(i) $\{T(\delta)\}$ is a proper operator in $H^{s, \gamma}(M)$ for each $\gamma$;

(ii) $\left\{T_{\gamma}(\delta)\right\}$ is elliptic for all $\gamma$ such that $T_{\gamma}$ is Fredholm.

We can ensure condition (ii) by using homotopies that preserve the conormal symbol:

$$
\sigma_{c}(T(\delta)) \equiv \sigma_{c}(T)
$$

whereas the amplitude ranges in the class of elliptic symbols.

As to condition (i), the possibility to ensure it can be derived from the following lemma.

Lemma 4.10. There is a homotopy

$$
g_{t}: T^{*} M \backslash\{0\} \rightarrow T^{*} M \backslash\{0\}
$$

in the class of homogeneous canonical transformations such that $g_{1}=g$ and $g_{0}$ commutes with translations along the t-axis for sufficiently large $t$ on the cylindrical end.

Let us introduce the simplest symmetry condition

$$
T_{0}(p)=T_{0}(-p)
$$

Then the conormal family

$$
g_{p}: T^{*} \Omega \rightarrow T^{*} \Omega, \quad \forall p \in \mathbb{R},
$$

of the canonical transformation $g$ satisfies the condition

$$
g_{p}=g_{-p} \quad \forall p \in \mathbb{R}
$$

Now if we cut away a neighborhood of infinity in the cylindrical end $T^{*} M$ and paste the resulting two manifolds with boundary with overlapping, we obtain the canonical transformation

$$
2 g: T^{*}(2 M) \backslash\{0\} \rightarrow T^{*}(2 M) \backslash\{0\}
$$

(See Fig,. 4.6, where the pasting is shown; the bottleneck is dashed as usual.). One can also prove that the symmetry condition ensures the smooth pasting of the amplitudes. 


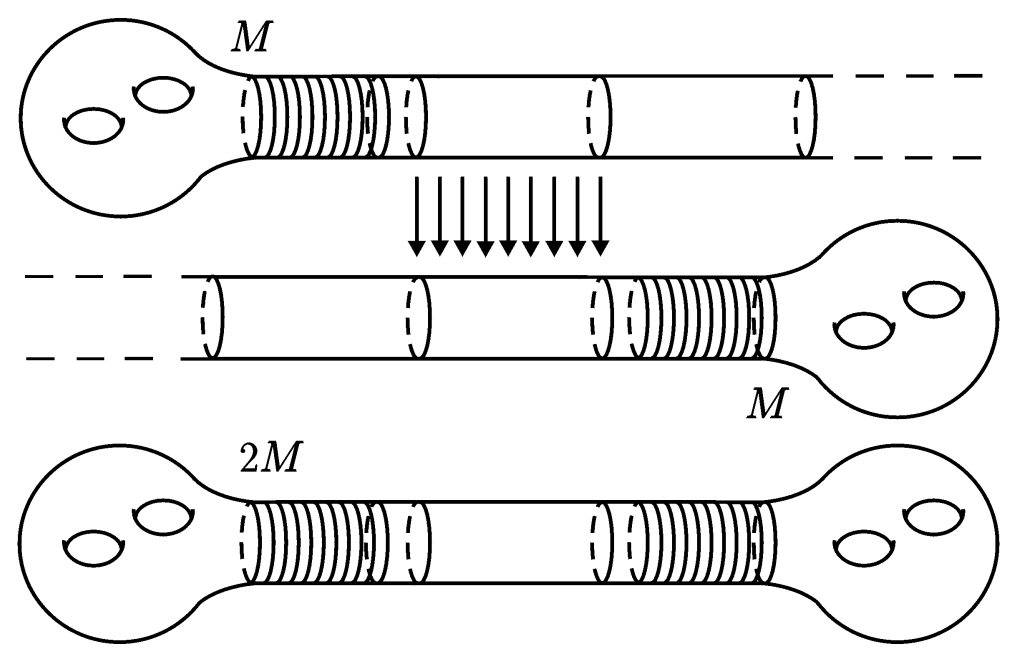

Figure 4.6:

Theorem 4.11. Let $T$ be a formally elliptic FIO on a manifold - $M$ with conical singularities satisfying the symmetry conditions (4.28). Then

$$
\text { ind } T_{\gamma}=\frac{1}{2}\left(\text { ind } 2 T-\sum_{-\gamma<\operatorname{Im} p_{j}<\gamma} m_{j}\right),
$$

for any $\gamma \geq 0$ such that the conormal symbol $T_{0}(p)$ is invertible on $\mathcal{L}_{\gamma}$. Here the $p_{j}$ are the poles of the family $T_{0}(p)^{-1}$, and the $m_{j}$ are their multiplicities. For $\gamma<0$, one has a formula similar to (4.30), with "+" instead of "- " on the sum of multiplicities.

Proof. Consider the modifications of operators in bottleneck spaces shown in Fig. 4.7 (surgery of Fourier integral operators) and 4.8 (another surgery). The geometric and analytic construction, with regard to the preceding, is completely similar to that for $\Psi$ DO. We have the modification diagram

$$
\begin{array}{ccc}
T_{\gamma} \oplus T_{-\gamma} \oplus B_{\gamma} & \stackrel{1}{\longleftrightarrow} & B_{\gamma} \oplus B_{-\gamma} \oplus B_{\gamma} \\
-1 \uparrow & \uparrow-1 \\
2 T_{\gamma} \oplus B_{\gamma} \oplus B_{-\gamma} & \stackrel{1}{\longleftrightarrow} & B_{\gamma} \oplus B_{\gamma} \oplus B_{-\gamma},
\end{array}
$$

which implies the desired result with regard to the fact that ind $B_{\gamma}=B_{-\gamma}=0$ and with the use of the general relative index theorem .

\subsubsection{Example}

In conclusion, we give an example of a canonical transformation $g$ for which there exists a FIO $T$ with conormal symbol satisfying the symmetry condition $T_{0}(p)=T_{0}(-p)$. 


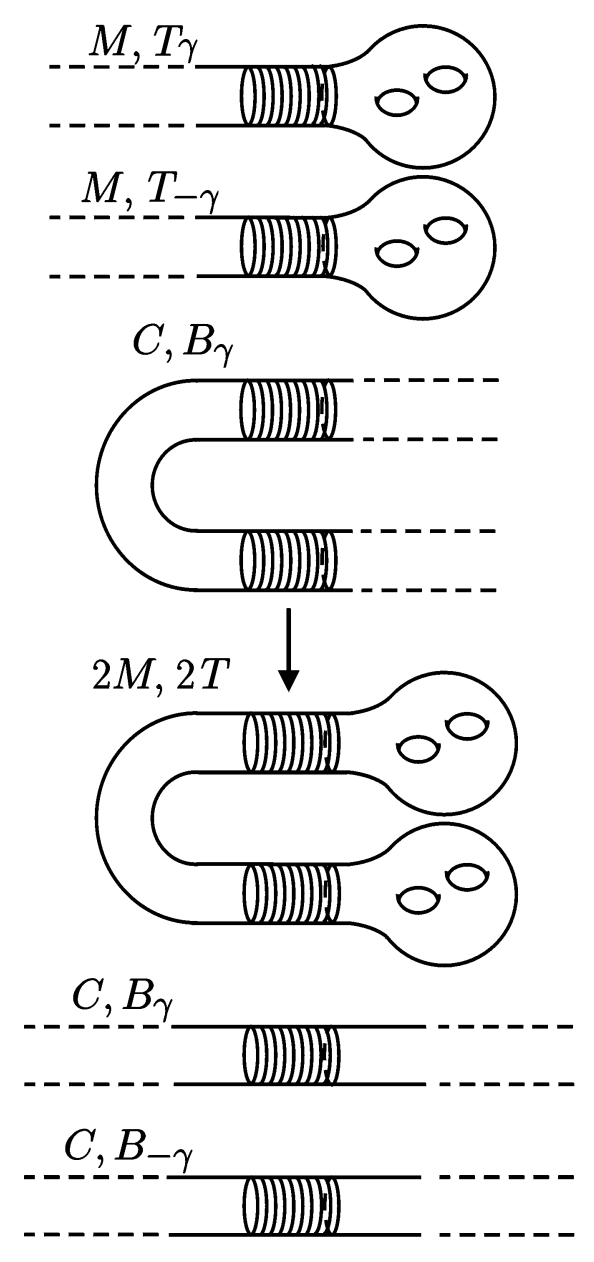

Figure 4.7:

More precisely, the transformation $g$ will be described only near the conical operator (only this is essential to the behavior of the conormal symbol). Let the base $\Omega$ of the one be the circle $S^{1}$ with mod $2 \pi$-coordinate $\omega$. We must specify the canonical transformation for large $t$ on the infinite cylinder $C=(-\infty, \infty) \times S^{1}$ with coordinates $(t, \omega)$. Let $p$ and $q$ be the coordinates dual to $t$ and $\omega$, respectively, and let

$$
g:(t, \omega, p, q) \mapsto\left(t^{\prime}, \omega^{\prime}, p^{\prime}, q^{\prime}\right)
$$

be specified by the generating function

$$
S\left(p, q, t^{\prime}, \omega^{\prime}\right)=\sqrt{p^{2}+q^{2}}
$$




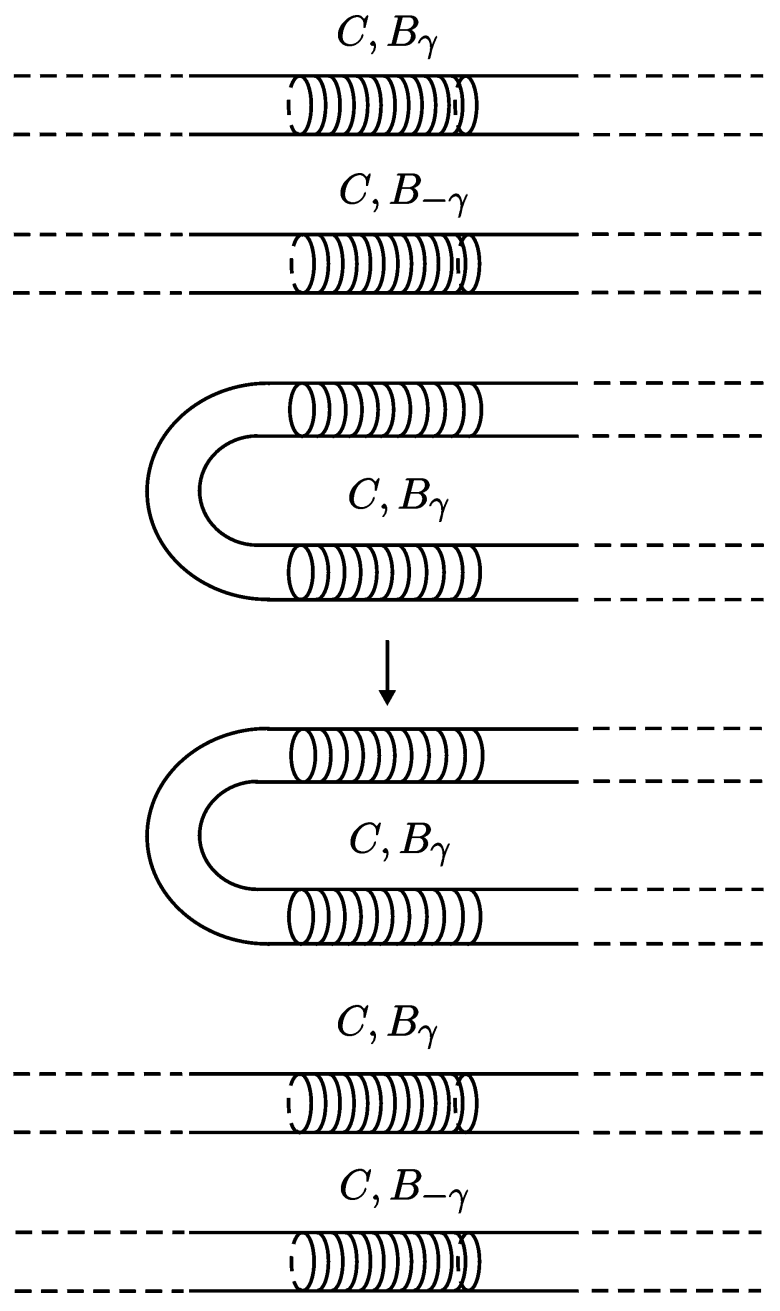

Figure 4.8:

according to the formulas

$$
\begin{aligned}
t & =t^{\prime}+\frac{\partial S}{\partial p}=t^{\prime}+\frac{p}{\sqrt{p^{2}+q^{2}}} \\
\omega & =\omega^{\prime}+\frac{\partial S}{\partial q}=\omega^{\prime}+\frac{q}{\sqrt{p^{2}+q^{2}}} \\
p^{\prime} & =p+\frac{\partial S}{\partial t^{\prime}}=p \\
q^{\prime} & =q^{\prime}+\frac{\partial S}{\partial \omega^{\prime}}=q .
\end{aligned}
$$


Expressing the primed variables via nonprimed, we see that the transformation is given by

$$
\begin{gathered}
p^{\prime}=p \quad t^{\prime}=t-\frac{p}{\sqrt{p^{2}+q^{2}}}, \\
\omega^{\prime}=\omega-\frac{q}{\sqrt{p^{2}+q^{2}}}, \quad q^{\prime}=q .
\end{gathered}
$$

The second line in (4.32) specifies the conormal family

$$
g_{p}: T^{*} S^{1} \rightarrow T^{*} S^{1}
$$

of $g$. One can readily see that the symmetry condition $g_{p}=g_{-p}$ holds. To construct some operator $T$ corresponding to $g$ in a local chart near the singular point, we must smooth the function (4.31). Let $\chi(\tau)$ be a smooth compactly supported function such that $\chi(0)=1$ and $\chi(-\tau)=\chi(\tau)$, and let $\tilde{\chi}(p)$ be its Fourier transform. The the function

$$
\dot{S}(p, q)=\sqrt{p^{2}+q^{2}} * \tilde{\chi}(p) \equiv \int_{-\infty}^{\infty} \tilde{\chi}(p-\eta) \sqrt{\eta^{2}+q^{2}} d \eta
$$

has the same asymptotics for large $|p|+|q|, p \in \mathbb{R}$, as the function (4.31) but is analytic in the entire $p$-plane. Moreover,

$$
\dot{S}(-p, q)=\dot{S}(p, q)
$$

Now we define an operator $T$ on $(-\infty, \infty) \times S^{1}$ by setting

$$
T=\exp \left\{i \dot{S}\left(-i \frac{\partial}{\partial t},-i \frac{\partial}{\partial \omega}\right)\right\} .
$$

The conormal symbol $T_{0}(p)$ of this operator has the form

$$
T_{0}(p)=\exp \left\{i S\left(p,-i \frac{\partial}{\partial \omega}\right)\right\}
$$

or

$$
T_{0}(p) \psi=\sum_{k=-\infty}^{\infty} e^{i[S(p, k)+\omega k]} \psi_{k},
$$

where the $\psi_{k}$ are the Fourier coefficients of $\psi$ in the system $\left\{e^{i \omega k}\right\}$ :

$$
\psi=\sum_{k=-\infty}^{\infty} e^{i \omega k}
$$

Obviously, $T_{0}(p)$ satisfies the symmetry condition 4.28). Note that this symbol has no singular points (poles of $T_{0}(p)^{-1}$ ), so only the topological term in the index formula survives. 


\section{References}

[1] M. Agranovich and M. Vishik. Elliptic problems with parameter and parabolic problems of general type. Uspekhi Mat. Nauk, 19, No. 3, 1964, 53-161. English transl.: Russ. Math. Surv. 19 (1964), N 3, p. 53-157.

[2] M. Agranovich. Elliptic boundary problems. In M.S. Agranovich, Yu.V. Egorov, and M.A. Shubin, editors, Partial Differential Equations IX. Elliptic Boundary Value Problems, number 79 in Encyclopaedia of Mathematical Sciences, 1997, pages 1-144, Berlin-Heidelberg. Springer Verlag.

[3] N. Anghel. An abstract index theorem on non-compact Riemannian manifolds. Houston J. of Math., 19, 1993, 223-237.

[4] M.F. Atiyah and R. Bott. The index problem for manifolds with boundary. In Bombay Colloquium on Differential Analysis, 1964, pages 175-186, Oxford. Oxford University Press.

[5] M.F. Atiyah. Global theory of elliptic operators. In Proc. of the Int. Symposium on Functional Analysis, 1969, pages 21-30, Tokyo. University of Tokyo Press.

[6] M. Atiyah, V. Patodi, and I. Singer. Spectral asymmetry and Riemannian geometry I. Math. Proc. Cambridge Philos. Soc., 77, 1975, 43-69.

[7] M. Atiyah, V. Patodi, and I. Singer. Spectral asymmetry and Riemannian geometry III. Math. Proc. Cambridge Philos. Soc., 79, 1976, 71-99.

[8] B. Booß-Bavnbek and K. Wojciechowski. Elliptic Boundary Problems for Dirac Operators. Birkhäuser, Boston-Basel-Berlin, 1993.

[9] A.P. Calderón. Boundary value problems for elliptic equations. Outlines of the Joint Soviet-American Symposium on Partial Differential Equations, Novosibirsk, 1963, 303-304.

[10] J.J. Duistermaat. Fourier integral operators. In Lect. Notes Courant Inst., 1973. New York.

[11] C. Epstein and R. Melrose. Contact degree and the index of Fourier integral operators. Math. Res. Lett., 5, No. 3, 1998, 363-381.

[12] B.V. Fedosov, B.-W. Schulze, and N. Tarkhanov. A Remark on the Index of Symmetric Operators. Univ. Potsdam, Institut für Mathematik, Potsdam, February 1998. Preprint N 98/4.

[13] P.B. Gilkey and L. Smith. The eta invariant for a class of elliptic boundary value problems. Comm. Pure Appl. Math., 36, 1983, 85-132. 
[14] P.B. Gilkey and L. Smith. The twisted index problem for manifolds with boundary. J. Diff. Geometry, 18, No. 3, 1983, 393-444.

[15] P.B. Gilkey. Invariance Theory, the Heat Equation and the Atiyah-Singer Index Theorem. Publish of Perish. Inc., Wilmington Delawaere, 1984.

[16] M. Gromov and H.B. Lawson Jr. Positive scalar curvature and the Dirac operator on complete Riemannian manifolds. Publ. Math. IHES, 58, 1983, 295-408.

[17] L. Hörmander. The Analysis of Linear Partial Differential Operators. III. SpringerVerlag, Berlin Heidelberg New York Tokyo, 1985.

[18] L. Hörmander. The Analysis of Linear Partial Differential Operators. IV. SpringerVerlag, Berlin Heidelberg New York Tokyo, 1985.

[19] Ch.-Ch. Hsiung. The signature and $G$-signarute of manifolds with boundary. J. Diff. Geometry, 6, 1972, 595-598.

[20] Ch.-Ch. Hsiung. A remark on cobordism of manifolds with boundary. Arch. Math., XXVII, 1976, 551-555.

[21] E. Leichtnam, R. Nest, and B. Tsygan. Local formula for the index of a Fourier integral operator. preprint math.DG/0004022, 2000.

[22] V.P. Maslov. Théorie des Perturbations et Méthods Asymptotiques. Dunod, Paris, 1972. French transl. from the Russian 1965 edition.

[23] R. Melrose. The eta invariant of pseudodifferential operators and families. Math. Research Letters, 2, No. 5, 1995, 541-561.

[24] A. Mishchenko, V. Shatalov, and B. Sternin. Lagrangian Manifolds and the Maslov Operator. Springer-Verlag, Berlin-Heidelberg, 1990.

[25] V.E. Nazaikinskii and B.Yu. Sternin. A remark on elliptic theory on manifolds with isolated singularities. Russ. Math. Dokl., 374, No. 5, 2000, 606-610.

[26] V.E. Nazaikinskii and B.Yu. Sternin. The index locality principle in elliptic theory. Funkts. Anal. Prilozhen., 35, No. 2, 2001, 37-52.

[27] V. Nazaikinskii, B.-W. Schulze, B. Sternin, and V. Shatalov. Spectral boundary value problems and elliptic equations on singular manifolds. Differents. Uravnenija, 34, No. 5, 1998, 695-708. English trans.: Differential Equations, 34, N 5 (1998), pp $696-710$.

[28] V. Nazaikinskii, B.-W. Schulze, and B. Sternin. The Index of Quantized Contact Transformations on Manifolds with Conical Singularities. Univ. Potsdam, Institut für Mathematik, Potsdam, August 1998. Preprint N 98/16. 
[29] V. Nazaikinskii and B. Sternin. Localization and surgery in index theory of elliptic operators. In Conference: Operator Algebras and Asymptotics on Manifolds with Singularities, 1999, pages 27-28, Warsaw. Stefan Banach International Mathematical Center, Universität Potsdam, Institut für Mathematik.

[30] V. Nazaikinskii and B. Sternin. Localization and surgery in the index theory to elliptic operators. Russian Math. Dokl., 370, No. 1, 2000, 19-23.

[31] V. Nazaikinskii and B. Sternin. Surgery of Manifolds and the Relative Index to Elliptic Operators. Univ. Potsdam, Institut für Mathematik, Potsdam, Juli 1999. Preprint N 99/17.

[32] R.S. Palais. Seminar on the Atiyah-Singer index theorem. Princeton Univ. Press, Princeton, NJ, 1965.

[33] B.A. Plamenevskij and G.V. Rozenblyum. On the index of pseudodifferential operators with isolated singularities in the symbols. Leningrad Math. J., 2, No. 5, 1991, 1085-1110. Translated from Russian.

[34] B.A. Plamenevskij and G.V. Rozenblyum. Pseudodifferential operators with discontinuous symbols: $K$-theory and the index formula. Funct. Anal. Appl., 26, No. 4, 1992, 266-275.

[35] G. Rozenblioum. Index formulae for pseudodifferential operators with discontinuous symbols. Ann. Global Anal. Geometry, 15, No. 1, 1997, 71-100.

[36] A. Savin, B.-W. Schulze, and B. Sternin. On the invariant index formulas for spectral boundary value problems. Differentsial'nye uravnenija, 35, No. 5, 1999, 705-714. [Russian].

[37] B.-W. Schulze, B. Sternin, and V. Shatalov. Structure rings of singularities and differential equations. In Differential Equations, Asymptotic Analysis, and Mathematical Physics, number 100 in Mathematical Research, 1996, pages 325-347, Berlin. Akademie Verlag.

[38] B.-W. Schulze, B. Sternin, and V. Shatalov. Differential Equations on Singular Manifolds. Semiclassical Theory and Operator Algebras, volume 15 of Mathematics Topics. Wiley-VCH Verlag, Berlin-New York, 1998.

[39] B.-W. Schulze, B. Sternin, and V. Shatalov. On general boundary value problems for elliptic equations. Math. Sb., 189, No. 10, 1998, 145-160. English transl.: Sbornik: Mathematics 189, N 10 (1998), p. 1573-1586.

[40] B.-W. Schulze, B. Sternin, and V. Shatalov. On the index of differential operators on manifolds with conical singularities. Annals of Global Analysis and Geometry, 16, No. 2, 1998, 141-172. 
[41] R.T. Seeley. Topics in pseudodifferential operators. In L. Nirenberg, editor, PseudoDifferential Operators, 1969, pages 167-305, Roma. C.I.M.E. Conference on pseudodifferential operators, Stresa 1968, Cremonese.

[42] R.E. Stong. Manifolds with reflecting boundary. J. Diff. Geometry, 9, 1974, 465-474.

[43] N. Teleman. The index of signature operators on Lipschitz manifolds. Publ. Math. IHES, 58, 1984, 39-78.

[44] A. Weinstein. Fourier integral operators, quantization, and the spectrum of a riemannian manifold. In Géométrie Symplectique et Physique Mathématique, number 237, 1976, pages 289-298. Colloque Internationale de Centre National de la Recherche Scientifique.

[45] A. Weinstein. Some questions about the index of quantized contact transformations. RIMS Kôkûryuku, 104, 1977, 1-14. 